University of Louisville

ThinkIR: The University of Louisville's Institutional Repository

Electronic Theses and Dissertations

8-2003

\title{
One bug, two drugs : A mathematical model of resistance dynamics in the ICU.
}

Susan L. Calcote White 1971-

University of Louisville

Follow this and additional works at: https://ir.library.louisville.edu/etd

\section{Recommended Citation}

White, Susan L. Calcote 1971-, "One bug, two drugs : A mathematical model of resistance dynamics in the ICU." (2003). Electronic Theses and Dissertations. Paper 1560.

https://doi.org/10.18297/etd/1560

This Master's Thesis is brought to you for free and open access by ThinkIR: The University of Louisville's Institutional Repository. It has been accepted for inclusion in Electronic Theses and Dissertations by an authorized administrator of ThinkIR: The University of Louisville's Institutional Repository. This title appears here courtesy of the author, who has retained all other copyrights. For more information, please contact thinkir@louisville.edu. 


\title{
ONE BUG, TWO DRUGS: \\ A MATHEMATICAL MODEL OF RESISTANCE DYNAMICS \\ IN THE ICU
}

\author{
By \\ Susan L. Calcote White \\ B.S., McNeese State University, 1992
}

\begin{abstract}
A Thesis
Submitted to the Faculty of the

Graduate School of the University of Louisville in Partial Fulfillment of the Requirements for the Degree of
\end{abstract}

Master of Arts

Department of Mathematics

University of Louisville

Louisville, Kentucky

August 2003 
ONE BUG, TWO DRUGS:

A MATHEMATICAL MODEL OF RESISTANCE DYNAMICS

IN THE ICU

\author{
By \\ Susan L. Calcote White \\ B.S., McNeese State University, 1992
}

A Thesis Approved on

July 17,2003

by the following Thesis Committee:

Thesis Director 


\section{ACKNOWLEDGMENTS}

I would like to thank my thesis advisor, Dr. Elizabeth Bradley, for the time and guidance she has given me over the past year. I greatly appreciate the opportunity she gave me to work on such an interesting project. I would also like to thank the other members of my thesis committee, Dr. Bingtuan Li and Dr. William R. Rising, for sharing their insightful comments with me. Many thanks to my parents, L.L. and Kay Calcote, who instilled in me a love of learning that has sustained me during my years in school. Finally, I would like to thank my husband, Daren. None of this would have been possible were it not for his encouragement and support. 


\title{
ABSTRACT \\ ONE BUG, TWO DRUGS: \\ A MATHEMATICAL MODEL OF RESISTANCE DYNAMICS IN THE ICU
}

\author{
Susan L. Calcote White
}

August 2003

Antibiotic resistance is a problem causing growing concern in the medical community, leading some to speculate that a return to the preantibiotic era is imminent. The problem of antibiotic resistance is particularly significant in the intensive care unit (ICU), due to the weakened immune responses of the patients and quantity of antibiotics administered. One theory proposes that the policy of cycling, or rotating, the antibiotics used in the ICU may minimize the development of resistance. Few clinical trials investigating the effects of cycling have been conducted, and many questions concerning the impact of cycling policies are unanswered at this point. In this thesis, we develop and analyze a mathematical model designed to examine resistance dynamics in the ICU in response to a cycling policy. The uncertainty analysis performed on the model evaluates the variability of the model outcome due to the uncertainty in estimating input values. The chosen method of analysis is Latin Hypercube Sampling (LHS). This uncertainty analysis is extended with the Latin Hypercube Sampling/Partial Rank Correlation (LHS/PRC) sensitivity analysis technique, which identifies the input variables that have the greatest effect on the model outcome. The analysis results show that the prediction imprecision of the model is quite high, leading us to conclude that the model's potential as an investigative tool cannot be fully realized until input values can be estimated with greater certainty. 


\section{TABLE OF CONTENTS}

PAGE

ACKNOWLEDGEMENTS ...........................................................ii

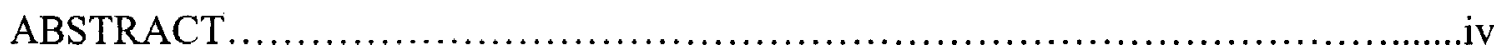

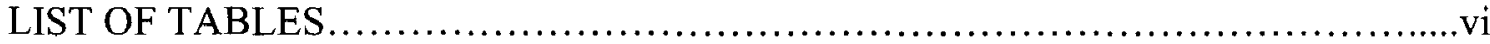

LIST OF FIGURES.............................................................

CHAPTER

I. INTRODUCTION ........................................................... 1

II. Pseudomonas Aeruginosa ......................................... 8

2.1 The Microbial Hyena............................................... 8

2.2 Antibiotic Treatment and Resistance...............................11

2.3 Fighting Infection in the ICU................................. 15

III. THE ONE BUG-TWO DRUG MODEL................................19

3.1 The Chemostat Model............................................20

3.2 The One Bug-Two Drug Model of the ICU..........................23

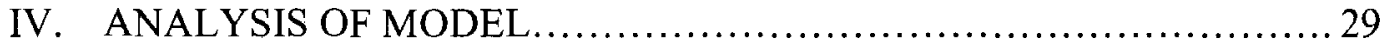

4.1 The LHS and LHS/PRC Analysis Techniques.......................... 29

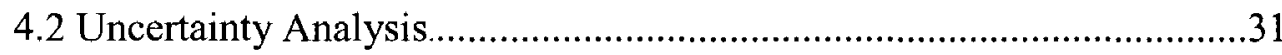

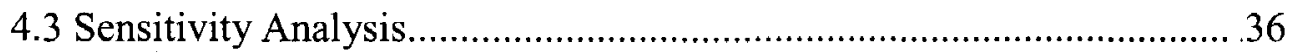

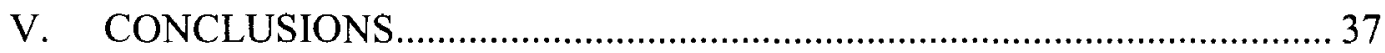

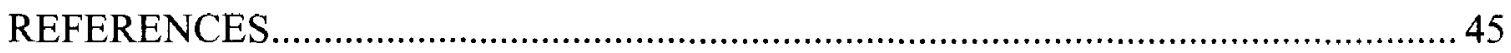

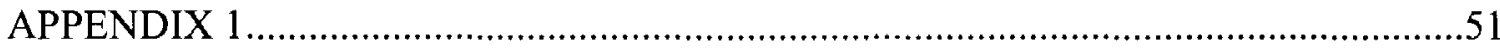

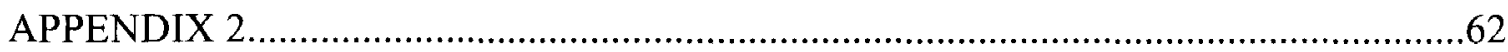

CURRICULUM VITAE 


\section{LIST OF TABLES}

TABLE

PAGE

1. Common Pathogenic Bacteria ...........................................

2. Origins of Resistance........................................................ 12

3. Mechanisms of Resistance................................................ 13

4. Parameters in the Chemostat Model.......................................... 21

5. Parameters in the One Bug-Two Drug Model............................27

6. Comparison of Simple Chemostat and ICU...................................28

7. Probability Distribution Functions for Input Variables......................................33

8. Descriptive Statistics for Outcome Variables at Time $t=480$ days...................35

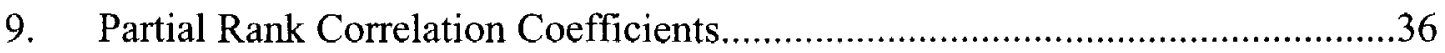

10. Comparison of Partial Rank Correlation Coefficients

with respect to $B_{r s}$ and $B_{s r}$ at Times $\mathrm{t}=360$ and $\mathrm{t}=480$ days....................... 41 


\section{CHAPTER I}

\section{INTRODUCTION}

"All the effects of nature are only the mathematical consequences of a small number of immutable laws."

Pierre-Simon De Laplace, Quoted in Men of Mathematics

The use of mathematical tools to investigate biological phenomena has undergone rapid growth in recent years, and by all accounts this trend is expected to continue. Ian Stewart writes in [2], "I predict - and I am by no means alone - that one of the most exciting growth areas of twenty-first-century science will be biomathematics." (p. xii) The current flourishing of biomathematics is usually attributed to the introduction of high-speed computing into the field. One example illustrating the usefulness of computers in applying mathematics to biology can be found in the study of animal coat patterns. In the $1950 \mathrm{~s}$, the great British mathematician Alan Turing suggested that mathematics could be used to analyze the coat patterns of animals [3]. He believed that equations could be found which characterize the processes by which coat patterns form. Over thirty years passed before Turing's idea came to fruition. In the late 1980s, James Murray, a mathematician at the University of Oxford, wrote a set of equations describing the aforementioned processes, solved the equations using a computer program, and transformed the solutions into images with the aid of computer graphics. Murray's results have been used to put forward a plausible theory explaining a phenomenon which has perplexed zoologists for years: why are some animals spotted and others striped?

Today, the fields of biology and mathematics are intersecting in diverse areas to produce groundbreaking results. Knot theory is used to study DNA, graph theory is used 
to develop phylogenetic trees detailing species evolution, and fractals are used to study circulatory, respiratory, and nervous systems in the body [4]. Although many sophisticated mathematical techniques are now available, some biological occurrences are still best idealized through the use of mathematical models consisting of systems of differential equations.

Differential equations are particularly appropriate in studying biological processes which occur with respect to time, such as the growth and decline of a population. The use of differential equations in population biology dates back over 300 years. In 1798, Malthus used a simple equation to predict population growth. This equation,

$$
\frac{d N}{d t}=K N,
$$

where $N$ is population density at time $t$ and $K$ is rate of reproduction per unit time, is sometimes called the Malthus law. Malthus applied this model to human population growth and deduced that the world's population would grow so large that humankind would eventually suffer mass starvation [5]. Pierre-François Verhulst, a Belgian mathematician [6], was an influential figure in the field in the nineteenth century. The equation

$$
\frac{d P}{d t}=P(\beta-\delta P),
$$

where $P$ is the total number of organisms in a population at time $t$, and $B$ and $\delta$ are the birth and death rates, respectively, of the population, is known as the logistic equation, or Verhulst's equation [7]. The "carrying capacity" in this equation is the ratio $\frac{\beta}{\delta}$. In the twentieth century, Alfred J. Lotka and Vito Volterra, who arrived at their results independently of each other, played significant roles in population biology. The equations which make up the classic deterministic model of a single predator-single prey system are called the Lotka-Volterra equations. These equations are

$$
\begin{aligned}
& \frac{d H}{d t}=H(a-\alpha P) \\
& \frac{d P}{d t}=P(-b+\beta H)
\end{aligned}
$$


where $H$ and $P$ are the populations of predator and prey, respectively, at time $t$, and $a$ is birth rate of prey, $b$ is death rate of predator, and $\alpha, \beta$ are parameters relating to species interaction [8]. This model has been extended for use in a system with multiple predators and prey.

Historically, even models which are somewhat inaccurate in representing realworld phenomena have proven to be useful in other ways. For example, in 1935, entomologist A.J. Nicholson and physicist V.A. Bailey proposed a model predicting the oscillations in the populations of two species which form a host-parasitoid pair $[5,6]$. The Nicholson-Bailey model, a system of two difference equations, is less stable than most host-parasitoid systems which occur in nature. However, by placing limitations on the model (for example, taking into account the fact that population growth is finite), the model becomes more stable. In this way, the model has been used to explore situations which have a stabilizing influence on host-parasitoid populations [5].

The model credited to Lotka and Volterra, shown above, has also been the target of criticism; it is often characterized as a naïve oversimplification of reality. The LotkaVolterra equations, for instance, do not take into account the age distribution of the population or the amount of resources available. But the fact that the model has been influential in biomathematics cannot be disputed. Volterra, in particular, was aware that the model was a simple one. He brought to the field of population biology his training in classical mechanics. His method was to begin with a few very simple hypotheses, find a mathematical representation of the problem, and gradually refine the model over time to approximate nature more closely [6].

Since the early twentieth century, population biology models have been used in the study of infectious diseases, both microparasitic (caused by viruses and bacteria) and macroparasitic (caused by larger organisms, such as worms). In 1911, Sir Ronald Ross used a mathematical model detailing the relation between mosquitoes, humans, and malaria to bolster his argument that controlling mosquito populations was the best way to curb the incidence of malaria [6]. Kermack and MacKendrick were also pioneers in this area. They proposed an early epidemic model [5] consisting of three ordinary differential equations: 


$$
\begin{aligned}
& \frac{d S}{d t}=-\beta I S \\
& \frac{d I}{d t}=\beta I S-v I \\
& \frac{d R}{d t}=v I .
\end{aligned}
$$

In this model, the population is divided into three subpopulations: those susceptible to the disease $(S)$, those infected by the disease $(I)$, and those "removed," which are no longer able to contract the disease $(R)$. The rate of disease transmission is denoted by $B$ and the rate of removal is $v$. The interpretation of the meaning of $v$ varies depending on the definition of $R$. For example, if population members are classified as "removed" because they have gained resistance to the disease, then $v$ is the rate at which resistance is gained; if population members enter class $R$ by death, then $v$ is death rate due to disease. Kermack and MacKendrick showed that this model was a close fit for data obtained from a 1906 plague which afflicted residents of Bombay. Kermack and MacKendrick are also credited with discovering the "threshold effect," the idea that a disease cannot become endemic unless the host population size is large enough.

Today, mathematical models consisting of differential equations are used to explore a variety of situations of interest to the medical community. Epidemic models are still in use; for example, models have been used to assess the consequences of intravenous drug use as it relates to the HIV transmission rate [9] and to predict the effect of vaccination policies on the public [5]. Models can also be used to study the effects of patient therapy; for example, the distribution of drugs throughout the body or glucoseinsulin dynamics in a diabetic patient can be accurately modeled. With the advent of high-speed computing, increasingly complex models are being formulated and numerically solved. This scenario offers unprecedented opportunity to the clinician in gathering information which could lead to improvements in patient care. In past years, health-care workers have made decisions based primarily on data obtained from clinical studies or empirical observations. However, the medical establishment is often reluctant to carry out experiments which may not meet ethical standards, or may be too costly 
and/or time-consuming. Hence, this data is not always readily available. If a model with good predictive ability can be constructed to investigate the dynamics of a medical problem, the solution of the model can be studied and the results can take the place of experimental data. The model is thus able to produce information that cannot be obtained by other means.

One serious problem facing the medical community today is the development of antibiotic resistance by pathogenic (disease-causing) bacteria. Certain patient groups are at increased risk for infection by resistant bacteria. ICU patients make up one particularly vulnerable group. These patients often have weakened immune systems due to underlying illness, coupled with natural barrier defenses being compromised through the use of invasive medical devices (IV's, catheters, surgical wounds, etc.). In some cases, it is impossible to treat patients infected with resistant bacteria, leading to an increased mortality rate.

The dilemma facing the medical community is that resistance develops primarily as a result of antibiotic use. The antibiotics provide the selective pressure allowing bacteria with resistance-conferring mutations to flourish, i.e., “... antibiotics sow the seed of their own potential downfall..." (Levy, [10], p.vii) The clinician is essentially caught in a catch-22: antibiotic treatment is ineffectual for those patients most in need of it, such as patients in the ICU. It has been postulated that resistance may be minimized by carefully controlled antibiotic policies. One theory proposes that a policy of "cycling," or rotating, the antibiotics used in the ICU may minimize resistance development by keeping the bacteria "confused." In other words, the mechanisms of resistance vary depending on the drug in use, so if the selective pressure allowing for the development of resistance is constantly changing, the bacteria cannot become resistant. Before the bacteria have a chance to develop resistance to a particular drug, the drug is removed from the environment and replaced with a different one.

The strategy of cycling seems promising in theory, but many questions are unanswered at this point. Is cycling effective in preventing resistance? If so, is it effective for all species of pathogenic bacteria? What is the optimal cycle length to prevent resistance? What combination of drugs should be cycled? What are the longterm effects of cycling? Clearly it is implausible to conduct clinical trials investigating so 
many different scenarios. Mathematical modeling is proving to be indispensable in exploring the effects of ICU cycling policies. If a model which examines the dynamics of resistance in the ICU can be constructed and its predictive ability is good, such questions can be easily answered by performing model simulations.

The output of a mathematical model of resistance dynamics must be matched with experimental data before it can be used to answer questions such as those listed above. Several hospitals have conducted clinical trials investigating the effects of cycling. One of these trials was recently performed at Hennepin County Medical Center (HCMC) in Minneapolis, MN. The antibiotics ciprofloxacin and piperacillin/tazobactam were rotated every four months in the ICU at HCMC. Many bacterial species, including Staphylococcus aureus and Pseudomonas aeruginosa, were collected from infected patients and tested for resistance throughout the study. The duration of the trial was 16 months ( 480 days), and the data set from the trial was collected in September 2001 by Dr. Dean Tsukayama, infectious disease specialist at HCMC. The data from this experiment will be discussed in Chapter 5 of this paper. Another clinical trial is currently being performed at Utrecht University Medical Center in Amsterdam; the results from this trial are not yet available.

The purpose of this thesis is to construct and evaluate a mathematical model that may be used to investigate the dynamics of resistance development in the ICU in response to a cycling policy. The model we present is a system of differential equations containing some of the elements of the influential population biology models presented earlier in this chapter, such as logistic growth (Verhulst) and the division of a population into subpopulations, where population members may move from one subpopulation to another (Kermack and MacKendrick). The model is designed to track the gain and loss of antibiotic resistance in a bacterial population when two drugs are cycled. The bacterial species chosen for the model in this paper is Pseudomonas aeruginosa and the drugs are ciprofloxacin and piperacillin/tazobactam; these choices were made so that the model could ultimately be compared with the data from the clinical trial performed at HCMC.

To accomplish the task, we merge biological principles, mathematical modeling, and computing capabilities. Chapter 2 deals primarily with the biology of the situation, with research into the properties of $P$. aeruginosa, ciprofloxacin, and 
piperacillin/tazobactam, and the unique challenges faced by health-care workers fighting bacterial infection in the ICU. Chapter 3 focuses on the mathematical modeling processes used to develop the model. The "chemostat" model is introduced in Chapter 3. A discussion of the equations used in the chemostat model is included, as well as the biological justifications for using this particular model as a basis for the model developed in this paper. We bring our computing capabilities to bear in Chapters 4 and 5. Chapter 4 describes the chosen method of analysis used on the model, Latin Hypercube Sampling, and presents the results of that analysis. In Chapter 5, we present a model simulation obtained through the use of a computer program and discuss the implications of the results found in Chapter 4 . We also discuss the difficulties encountered in matching the model output to experimental data, and conclude with a discussion of the directions this work may take in the future. 


\section{CHAPTER II}

\section{Pseudomonas Aeruginosa}

"It, as with the wolf-like carnivore, is a pathogen characterized by hemorrhage and necrosis, yet like the cowardly part of the hyena, Pseudomonas frequently colonizes the human host, waiting for an opportunity to spring."

Robert B. Fick, Jr., Pseudomonas aeruginosa the Opportunist: Pathogenesis and Disease

\subsection{The Microbial Hyena}

The first published scientific study of Pseudomonas aeruginosa appeared over 120 years ago. A physician in France, Dr. Chauvel of Val-de-Grâce, noticed that the bandages used on some of his patients had an unusual blue-green stain. He gave the used bandages to Carle Gessard, a French pharmacist, who began tests to determine the cause. Gessard identified the pigment as pyocyanine; "pyo" is a prefix meaning pus, and "cyanine" is a type of blue dye. The pyocyanine was being emitted by an organism that Gessard described as colorless, globular, aerobic, and very mobile. Gessard published his results in an article entitled "On the blue and green coloration of bandages" in 1882 $[12,13]$. Gessard's paper dealt almost exclusively with the chemical properties of the pigment-producing organism that is known today as Pseudomonas aeruginosa. In the years since its discovery, $P$. aeruginosa has gone by several different names: Bacillus pyocyaneus, Pseudomonas polycolor, Bakterium aeruginosa, and Pseudomonas pyocyaneus (note that several of these names indicate the presence of the pyocyanine discussed by Gessard).

Pseudomonas aeruginosa is a bacterial species. Bacteria are microscopic unicellular organisms which reproduce by cell division. Bacteria are present both in the 
natural world and in living beings. The average human being, for example, is host to approximately $10^{14}$ bacteria in the form of intestinal and skin flora [10]. Clearly, then, it is not the case that all bacteria cause disease. However, some bacteria, when present in the body as a sufficiently large colony, are disease-causing. These bacteria are called pathogenic.

Bacteria are classified into two groups: Gram-positive and Gram-negative. This classification is made according to whether a bacterium retains a violet stain used during a procedure called Gram's method. Gram's method is named for the Danish physician Hans Christian Gram (1853-1938). In the latter part of the $19^{\text {th }}$ century, Gram created a technique whereby he first stained a bacterial specimen with a deep purple dye and then washed it. Those specimens which retained the color after washing were classified as Gram-positive (or stain-positive); those which did not were Gram-negative. $P$. aeruginosa falls into the second category. A list of several common pathogenic bacteria is shown in Table 1.

Table 1 Common Pathogenic Bacteria

\begin{tabular}{ll}
\hline Gram-positive & Gram-negative \\
\hline Streptococcus pneumoniae & Escherichia coli \\
Staphylococcus aureus & Hemophilus influenzae \\
& Neisseria gonorrhoeae \\
& Pseudomonas aeruginosa \\
\hline
\end{tabular}

The Gram-positive/Gram-negative classification is significant in that it gives important information about the cell wall of a bacterium. The Gram-positive bacteria have a single cell wall, while the Gram-negative bacteria have a cell wall consisting of three layers. The dye used in Gram's method is thus able to penetrate the outer layer of a Gram-positive specimen. This distinction between Gram-positive and Gram-negative bacteria must be taken into account when studying the effectiveness of antibiotics against different types of pathogenic bacteria; this will be discussed in more detail in Section 2.2.

The $P$. aeruginosa species is a member of the family Pseudomonadaceae. This family of rod-shaped, Gram-negative bacteria, called pseudomonads, is a large group containing a variety of species. The pseudomonads have been known to grow in both 
aerobic and anaerobic conditions, and are found in such diverse environments as water, soil, decaying matter, sewage, insects, plants, and animals. Four species of this family are known to be pathogenic to humans throughout the world; they are $P$. aeruginosa, $P$. mallei, $P$. cepacia, and $P$. pseudomallei. Only $P$. aeruginos $a$ and $P$. cepacia are present in significant numbers in the United States [11]. Certain species of pseudomonads, $P$. aeruginosa in particular, have plasmids which allow for the development of special features such as antibiotic resistance [14]. Plasmids are units of DNA within a cell that are independent of the chromosomal DNA and can transfer genetic material from cell to cell. Plasmids directly contribute to the ability of bacteria to survive within their environment by facilitating the evolution of the bacteria, the end result being that the bacteria are better able to deal with harmful substances within that environment.

Prior to $1947, P$. aeruginosa was not considered a serious threat by the medical community, with only 91 cases of human infection described in the literature. However, the second half of the century saw a sharp increase in the number of cases of Pseudomonas infections, accompanied by a rising mortality rate. What could have caused such a change? Pseudomonas aeruginosa is often described as an "opportunistic" pathogen [11,15] because Pseudomonas infections occur almost exclusively in the immunocompromised host. With the health care advances of the mid to late twentieth century, the life expectancies of these patient groups began to increase, thereby increasing the numbers of those most vulnerable to Pseudomonas infections.

Although Pseudomonas poses no threat to the healthy host, those at risk face the possibility of quite serious complications. P. aeruginosa, as befits a pathogen known as opportunistic, can "infect virtually any part of the body, causing endocarditis, respiratory infections, bacteremia, central nervous system infections, ear and eye infections, bone and joint infections, and infections of the urinary tract, soft tissues and skin, and gastrointestinal tract" [15] (p. 297). Because $P$. aeruginosa preys exclusively on the weak and vulnerable among us, it has been called a "microbial hyena" [11].

Certain hospital patient groups are particularly vulnerable to Pseudomonas infections. One such group is cystic fibrosis patients. Cystic fibrosis (CF) is the most common lethal genetic disease among Caucasians [11,15], and those suffering from CF often deal with chronic respiratory infections caused by $P$. aeruginosa. In these patients, 
antibiotic treatment fails to eradicate the infection due to the development of antibiotic resistance. The eventual result is impaired lung function and an increased mortality rate. The European Epidemiologic Registry of Cystic Fibrosis reported in 2001 that $54.2 \%$ of children with $\mathrm{CF}$ between the ages of 6 and 12 and $78.8 \%$ of adults with $\mathrm{CF}$ over the age of 18 were afflicted with Pseudomonas infections [16].

$P$. aeruginosa infection is the leading cause of death among burn patients, with mortality rates approaching $77 \%$ [15]. In fact, burn patients were among the first patient groups in which the severity of the pathogenicity of Pseudomonas aeruginosa was recognized and studied. The burned mouse model was developed in 1975 for this purpose, and today it remains a well-known model used to study Pseudomonas infections in burn patients.

A third group likely to suffer from $P$. aeruginosa infection are those critical care patients in the ICU. While a healthy patient is protected by mucosal and skin barriers which present an impediment to bacteria attempting to enter the body, those barriers are often breached in an ICU patient through the use of invasive devices such as ventilators, catheters, and intravenous drugs [17]. In addition, the ICU patient is immunocompromised in many cases, due to malnutrition and/or serious underlying illness.

\subsection{Antibiotic Treatment and Resistance}

The first line of defense against bacterial infection is antimicrobial therapy, i.e., the use of antibiotics, but antimicrobial-resistant pathogens are becoming increasingly common today. The problem of resistance was recognized even during the early days of antibiotic use. Alexander Fleming, the bacteriologist who discovered penicillin in 1928, was able to produce mutant bacteria in the laboratory that could not be killed by penicillin. He cautioned the medical community against the misuse of antibiotics, warning that such misuse would result in resistant strains of bacteria [10]. His words have proven prophetic with the passage of time. Today, the problem of resistant bacteria has reached such proportions that the journal Archives of Internal Medicine recently published an article with the startling subtitle "The preantibiotic era has returned" [18]. 
Antibiotics are substances produced by microorganisms (as opposed to being man-made) which are able to kill or inhibit the growth of bacteria. When antibiotics first came into use, they were considered nothing short of miraculous. Here at last was a "magic bullet" which was able to target and eradicate pathogenic bacteria. However, at present, even bacteria which have been susceptible to every known antimicrobial agent for years are developing resistance $[10,19]$. The evolutionary law of survival of the fittest applies to the microscopic world as well as the macroscopic; with the widespread use of antibiotics, the resistant bacteria are rapidly gaining an evolutionary advantage over the susceptible.

The origins of antibiotic resistance are varied (see Table 2). One way in which a strain of bacteria may become resistant is through genetic mutation in the chromosome of the bacteria. In some cases a single mutation causes resistance to arise; in other cases a less likely sequence of mutations must occur.

Table 2 Origins of Resistance

\begin{tabular}{l}
\hline Spontaneous genetic mutation \\
Gene exchange by plasmids \\
Latent resistance \\
Emergence of resistant subpopulations \\
\hline
\end{tabular}

Another way in which a bacterial strain may become resistant is through the exchange of genetic material. Plasmids, those non-chromosomal pieces of DNA briefly discussed in Section 2.1, play a vital role here. Plasmids are able to lose old genes or acquire new ones, and these genes sometimes code for antibiotic resistance. Plasmids can transfer not only from one bacterium to another within a colony, but also from one bacterial species to another. There are even reported cases of the transfer of genetic information between Gram-negative and Gram-positive bacteria [19]. A third way in which resistance may occur is known as latent resistance. Here, the chromosome of an organism contains inactive genes which confer resistance once they become active. The inactive genes are more likely to become active in the presence of antibiotic. Finally, some bacterial species contain a small resistant subpopulation, which becomes dominant once an antibiotic has killed the susceptible portion of the population. No matter the 
method by which bacteria acquire a defense mechanism, once acquired the bacteria may become resistant to an entire class of antibiotics, rather than just a single drug. Furthermore, in each of the methods described, it is the use of antibiotics which provides selective pressure favoring the development of resistance $[10,19,20]$.

The treatment of $P$. aeruginosa infection is a notoriously difficult task for the clinician. Mortality rates from such infections are high, sometimes exceeding $40 \%$, and $P$. aeruginosa has demonstrated an ability to rapidly acquire resistance [15,21]. Giamarellou and Antoniadou assert in [21] that "... steadily increasing resistance rates are reported worldwide, rendering the end of antibiotics for $P$. aeruginosa an approaching reality." (emphasis in original) The choice of antibiotic for any particular patient is dependent on several factors, including the site of infection in the body. Since $P$. aeruginosa is able to infect so many different areas of the body, it is imperative that physicians have a diverse group of antipseudomonal antibiotics from which to choose. In fact, several different classes of antibiotics have achieved some measure of success in fighting $P$. aeruginosa, including $\beta$-lactams, aminoglycosides, polymyxins, and quinolones $[15,22]$. We restrict our focus in this paper to two of these: the $\beta$-lactams and the quinolones. The mechanisms of resistance displayed by $P$. aeruginosa in response to these two classes of antibiotics are shown in Table 3.

Table 3 Mechanisms of Resistance

\begin{tabular}{|c|c|}
\hline \multirow[t]{3}{*}{$\beta$-lactams } & Gram-negative outer membrane \\
\hline & Production of $\beta$-lactamase \\
\hline & Alteration of penicillin-binding proteins \\
\hline \multirow[t]{3}{*}{ Quinolones } & Gram-negative outer membrane \\
\hline & Efflux \\
\hline & Alteration of DNA gyrase \\
\hline
\end{tabular}

An antibiotic must reach its target to be effective, and this target site varies from one antibiotic to another, as does the mechanism of resistance the bacteria develop to attempt to inactivate the antibiotic. The $\beta$-lactam antibiotics, in general, must reach the cell wall to be successful in killing or inhibiting the growth of the bacterium. Here $P$. aeruginosa, as a type of Gram-negative bacteria, has an edge over the Gram-positive 
bacteria in one particular mechanism of resistance. The drug must pass through an outer membrane that is present only in Gram-negative bacteria. The ability of the drug to cross the membrane is further compromised by mutations which may occur in the bacteria, making this membrane even less permeable. Another mechanism of resistance to $\beta$ lactams which has developed is the production of $\beta$-lactamase by the bacteria. $\beta$ lactamase is an enzyme which inactivates the $\beta$-lactam. To address the problem of $\beta$ lactamase production, clinicians often combine a $\beta$-lactam antibiotic with a $\beta$-lactamase inhibitor during the course of therapy [23]. The $\beta$-lactamase inhibitor, while itself not bactericidal, does prevent the $\beta$-lactamase from rendering the $\beta$-lactam ineffective. For example, the antibiotic piperacillin, a penicillin derivative, is often combined with the $\beta$ lactamase inhibitor tazobactam, with generally good results $[22,24,25,26]$. However, as one might expect, some $\beta$-lactamases are becoming resistant to $\beta$-lactamase inhibitors. A third way in which $P$. aeruginosa may gain resistance to a $\beta$-lactam antibiotic is by altering the penicillin-binding proteins (PBP's) on its surface. As a result of this change, the drug is no longer able to bind to its target.

Quinolones primarily target DNA gyrase in Gram-negative bacteria [27], interfering with the ability of the bacteria to reproduce by inhibiting the replication of its DNA. This mechanism of action is peculiar to the quinolones among all antibiotic classes [28]. The fluoroquinolones are a relatively new class of quinolones which are particularly effective against Gram-negative bacteria [29]. Ciprofloxacin, a fluoroquinolone, is the most powerful quinolone against $P$. aeruginosa $[15,21,28]$. Since the quinolones target a different site than the $\beta$-lactams, predictably, the mechanisms of resistance are different as well, with one exception. The mechanism of resistance that is common to both $\beta$-lactams and quinolones is the Gram-negative outer membrane. Once again, the drug must cross this membrane and mutations in the bacteria may make that difficult. A second mechanism of resistance that $P$. aeruginosa may develop against the quinolones is called efflux. In this case, the efflux mechanism, which is positioned in the cytoplasmic membrane, pumps the drug out of the cytoplasm before it can complete its task. Finally, $P$. aeruginosa may become resistant by altering its DNA gyrase, the target of the quinolones. The result is the same as with the $\beta$-lactams and the altered PBP's; the quinolone is not able to bind to its target. 
Although this section has focused primarily on one type of bacteria and two antibiotic classes, the problem of resistance is widespread, leading to international concern. This is not just the problem of individual hospitals, but a serious public health issue $[18,30]$. Unfortunately, treatment and resistance go hand-in-hand; the "antibiotic paradox" described by Levy in [10] is that resistance to antibiotics is actually a product of treatment with antibiotics. So it is that we look to our treatment policies to minimize resistance. Some strategies are well-known and undisputedly effective, such as ensuring that the patient completes the full course of antibiotic treatment and that the clinician prescribes antibiotics only when medically necessary. In addition, many recognize the need for well-defined antibiotic policies which govern the patterns of antibiotic use [31], but it is unclear exactly which of these strategies will work [32].

\subsection{Fighting Infection in the ICU}

With the exception of some CF patients, most of those fighting Pseudomonas infections acquired the infection while in the hospital. These hospital-acquired, or nosocomial, infections, occur frequently with $P$. aeruginosa because this bacterial species preys almost exclusively on the immunocompromised host, a population which is likely to be hospitalized. Hospital-wide, $P$. aeruginosa is the second most common Gramnegative nosocomial pathogen; only E. coli (Gram-negative) and S. aureus (Grampositive) occur more frequently [15]. Nosocomial infections due to resistant bacteria are particularly damaging to both hospitals and patients. A recent study found that a confirmed hospital-acquired infection imposes on the hospital an increased cost of $\$ 15,275$ per patient [33]. If the patient is infected with resistant bacteria, his/her hospital stay will likely be longer and thus more costly to the hospital; the hospital stay of such a patient is approximately 20 days longer than the stay of a similar patient infected with susceptible bacteria [34].

The ICU is a clinical unit which faces a particularly daunting task in fighting any type of bacterial infection. Here resistance rates are known to be higher than in any other area of the hospital [19,21], and antimicrobial-resistant pathogens may enter the ICU environment by several pathways unique to the ICU. Critical care patients sometimes require immediate treatment, so that health care workers are not able to properly disinfect 
their hands before administering treatment. This may result in antimicrobial-resistant pathogens crossing from patient to patient or being carried into the ICU from the outside environment. Critically ill patients are often transferred from one hospital to another, and some patients unknowingly carry resistant bacteria acquired from the facility from which they are transferred [17]. While antimicrobial-resistant pathogens are more likely to be present in the ICU, a typical ICU patient is more likely to become infected with such pathogens during his/her stay in the ICU than a patient in another area of the hospital. In addition to the immunocompromised status and weakened natural defenses of ICU patients discussed in Section 2.1, these patients are more likely to have previous exposure to antibiotics, which is perhaps the single greatest factor predisposing a patient to antibiotic resistance.

Not surprisingly, then, $P$. aeruginosa is one of the significant pathogens in the ICU $[18,35]$, where the compromised immune systems of the patients and ease with which $P$. aeruginosa develops resistance combine to make $P$. aeruginosa infection a formidable enemy. Recently released data from the National Nosocomial Infections Surveillance (NNIS) System shows that $P$. aeruginosa isolates collected in the ICU display resistance to five commonly used antibiotics, with resistance rates ranging from $13.9 \%$ to $37.8 \%$, depending on the antibiotic [36]. Resistance rates are sometimes much higher in a single hospital or particular geographic region [17,35,37]. The types of infection caused by $P$. aeruginosa in the ICU are usually either ventilator-associated pneumonia or catheter-associated urinary tract infections [17]. Although pneumonia may be caused by bacteria other than $P$. aeruginosa, those with this singular type of pneumonia are more likely to suffer from multiple organ failure and death [37]. Mortality rates can exceed $50 \%$ [21].

Many strategies have been proposed to fight nosocomial infection in the ICU. Some strategies do not involve a change in antibiotic policy, such as stricter adherence to guidelines concerning hand-washing and the use of gloves, gowns, and face masks by health care workers, as well as minimizing the number of hospital personnel which move in and out of the ICU [17]. Another strategy proposes the isolation of patients infected with resistant bacteria [19]. While these strategies are likely to reduce the frequency of nosocomial infection, the use of antibiotics will remain a necessity in many cases. 
However, there are no antimicrobials in use today that have not met with resistance [20]. We cannot depend on the discovery of new antibiotics to overcome the problem of resistance, as a tremendous amount of time (almost 20 years [23]) and money ( $\$ 100$ to $\$ 350$ million [20]) are required to bring a new drug from discovery to market in the United States.

Thus the medical community searches for a policy governing antibiotic use which will maximize the effectiveness of the antibiotics in use today, while minimizing the development of resistance in bacteria. Lepper, et al. [38] and Lesch, et al. [39] have presented data suggesting that when a hospital has an antibiotic control policy in place, resistance rates are reduced. However, Lesch argues that it is crucial that the ICU be studied separately from other units of the hospital; else unit-specific trends in antimicrobial use and resistance may not be recognized. In a study performed by the Centers for Disease Control and Prevention (CDC), the authors conclude that in monitoring antibiotic resistance in hospitals, antibiotic use and resistance must be observed jointly [40]. In both [39] and [40], the authors argue that a complex model is necessary to confirm a correlation between use and resistance of antibiotics.

In the ICU, it is often necessary to begin antibiotic treatment immediately, without waiting for laboratory results [41]. This type of therapy is known as empiric therapy, and the antibiotic used in such a situation is informally referred to as the "go-to" antibiotic in the ICU. The go-to antibiotic must have a broad spectrum of activity; i.e., it must be active against a wide variety of pathogenic bacteria. It has been suggested that if several go-to antibiotics are routinely rotated, the development of resistance will be minimized because the selective pressure favoring certain types of resistance will be periodically removed when another antibiotic is put into place. This policy is known as "cycling" antibiotics [42]. For example, if two antibiotics from different classes are routinely rotated (say, a $\beta$-lactam and a quinolone), then, theoretically, the mechanisms of resistance developed by the bacteria to fight the $\beta$-lactam will leave it susceptible to the quinolone, and vice versa. Essentially, the bacteria are "confused" and as soon as they begin to develop a mechanism of resistance, that mechanism becomes useless. Note that we make the assumption that in the absence of a specific antibiotic, the bacteria susceptible to that antibiotic will out-compete the bacteria resistant to the antibiotic. This 
is because resistance to antibiotics imposes a fitness cost on bacteria [43], so we may assume that in the absence of an antibiotic, the susceptible, or "wild-type," bacteria will dominate the bacterial population.

Raymond, et al., in [44], present the results of a study comparing two years in an ICU at the University of Virginia Health Sciences Center. During the first year, no antibiotic control policy was in place, but during the second year, empirical antibiotics were rotated quarterly. They found that mortality rates decreased, as did the number of antimicrobial-resistant infections, although the average length of hospital stay did not change. The authors conclude that the results seem promising, and further study is necessary.

The policy of cycling antibiotics has its critics, as well. Bonhoeffer, Lipsitch, and Levin [31] argue that it is always preferable to treat equal proportions of infected patients with different drugs simultaneously (in the case of two drugs, they call this 50-50 treatment) rather than cycling each drug on and off. The ideal time period for cycling drugs is one which selects the antibiotic with the smallest amount of resistance in the patient population at a given time. But the point in time at which the amount of resistance is at a minimum is when the drug is first cycled into use. So, theoretically, the best cycling policy is one which switches back and forth between the drugs with each new patient, which is identical to 50-50 treatment. It should be noted that this argument is based on the results of a mathematical model, rather than experimental data, and is not specific to the ICU.

While it remains unclear whether cycling antibiotics is the solution, it is certain that further study is necessary. Several guidelines for this study have been presented in this section; specifically, (1) the ICU should be studied separately from other areas in the hospital, (2) antimicrobial use and resistance should be jointly observed, and (3) a mathematical model is necessary to confirm a correlation between use and resistance of antibiotics when examining an antibiotic control policy. In the following chapter, we present a mathematical model which meets these three specifications; it is designed to examine the dynamics of a bacterial population in the ICU when two antibiotics are cycled. 


\section{CHAPTER III \\ THE ONE BUG-TWO DRUG MODEL}

"... it is the view in this work that studying the chemostat is much like studying the pendulum in physics; the precise assumptions for the pendulum model are not met, but there is much to be learned about oscillations by studying it."

Hal Smith \& Paul Waltman,

The Theory of the Chemostat: Dynamics of Microbial Competition

In this chapter, we present a mathematical model, based on the chemostat, which was designed to assess the dynamics of bacterial population in an ICU which has implemented a cycling policy that switches back and forth between two antibiotics as the empiric therapy drug of choice. The model consists of six ordinary differential equations which track the number of bacteria in the subpopulations that are (i) susceptible to both drugs, (ii) resistant to drug 1 and susceptible to drug 2, (iii) susceptible to drug 1 and resistant to drug 2, and (iv) resistant to both drugs, as well as (v) amount of drug 1 in the ICU, and (vi) amount of drug 2 in the ICU, at any given time. Since the model examines one particular type of bacteria displaying various degrees of resistance to two antibiotics, we call it the "one bug-two drug" model. For the purposes of the model analysis in Chapter IV and comparison with experimental data (see Chapter V), the "bug" is assumed to be $P$. aeruginosa, drug 1 is ciprofloxacin, and drug 2 is piperacillin/tazobactam. However, the model can be easily adapted to other bacteria and antibiotics by changing the parameter values. 


\subsection{The Chemostat Model}

The chemostat is a simple piece of laboratory equipment which plays an important role in the study of the growth of microorganisms. Many ecological models are based on the chemostat; for instance, the chemostat has been used to model wastewater treatment problems [45]. The chemostat has gained widespread acceptance as a useful model in ecology [46,47]. F.M. Williams writes in [48] that the "chemostat is the best laboratory idealization of nature for population studies. It is a dynamic system with continuous material inputs and outputs, thus modeling the open system character and temporal continuity of nature." (p. 200)

The chemostat, as described by Smith and Waltman in [45], consists of three connected devices. The first container is called the feed bottle. It holds a solution containing all of the nutrients which are necessary for the growth of the microorganism. The second container is the bacterial growth chamber, or culture vessel. The third container is the overflow vessel. The nutrient solution is pumped out of the feed bottle and into the culture vessel at some fixed rate. At the same time, the culture vessel has an outflow valve which allows its contents to flow out of the culture vessel and into the collection vessel at the same rate. Since inflow and outflow are always equal, the volume of the culture vessel is constant. The culture vessel contains both nutrient and microorganisms, so some microorganisms, as well as a small amount of nutrient, will be removed from the culture vessel during outflow. It is assumed that the culture vessel is kept well stirred, and all parameters affecting the growth of the microorganisms (e.g., temperature, $\mathrm{pH}$ level) are fixed. With these assumptions in place, bacterial growth depends only on the amount of nutrient in the culture vessel at any given time.

Smith and Waltman give two ordinary differential equations to describe the rates of change of both nutrient and organism in the culture vessel. Simply put, the equations describe the rate of change of nutrient as inflow minus outflow minus depletion due to bacterial growth, and the rate of change of organism as growth minus outflow. It is assumed in the chemostat model that bacterial growth is directly proportional to nutrient availability only up to a limiting value [5]. Since the growth rate is a saturating function of nutrient concentration, this rate typically incorporates Michaelis-Menten kinetics. The 
growth rate is defined as $\frac{m S}{a+S}$, where $S$ is the concentration of nutrient, $m$ is the maximum growth rate, and $a$ is that value of $S$ for which the growth rate is one-half of the maximum. The equation defining the growth rate of the bacteria,

$$
\text { (growth rate) }=\frac{m S}{a+S}
$$

is known as the Monod equation $[45,46]$. The following are the differential equations adapted from [45]. Parameters are defined in Table 4.

$$
\begin{aligned}
& \frac{d S}{d t}=\frac{F}{V} S_{o}-\frac{F}{V} S-\frac{1}{\gamma} \frac{m S}{a+S} x \\
& \frac{d x}{d t}=\frac{m S}{a+S} x-\frac{F}{V} x \\
& S(0) \geq 0, x(0)>0 .
\end{aligned}
$$

Table 4 Parameters in the Chemostat Model

\begin{tabular}{cll}
\hline Parameter & Units & Description \\
\hline$S$ & mass/volume & Concentration of nutrient in culture vessel at time t \\
$x$ & mass/volume & Concentration of organism in culture vessel at time t \\
$S_{o}$ & mass/volume & Concentration of nutrient in feed vessel \\
$\gamma$ & none & Yield constant reflecting nutrient depletion due to \\
& & bacterial growth \\
$m$ & 1/time & Maximum growth rate of organism \\
$a$ & mass/volume & Michaelis-Menten constant \\
$F$ & volume/time & Volumetric flow rate \\
$V$ & volume & Volume of culture vessel \\
\hline
\end{tabular}

Using the simple chemostat containing only nutrient $S$ and a single species $x$, many different scenarios may be investigated by introducing additional elements into the chemostat environment. For example, a researcher may introduce two different organisms $x_{1}$ and $x_{2}$ which compete for nutrient $S$ into the culture vessel in order to study the competition between the two organisms. The model of the chemostat in this case 
would consist of three differential equations, one each for $S, x_{1}$, and $x_{2}$. Another variation of the chemostat may involve nutrient $S$ and organism $x$, as well as two additional predators on $x$, say, $y$ and $z$. The model of this chemostat contains four differential equations. (The equations for these two scenarios are not shown here but can be found in [45].)

Lenski and Hattingh propose another variation of the chemostat in [49]. They present a model which investigates the dynamics of two species competing for nutrient in the presence of a substance which inhibits the growth of one of the species but not the other. The growth-inhibiting substance is input into the environment from an external source, as opposed to being produced by one of the competing species. The former scenario is called an external inhibitor problem, while the latter is an internal inhibitor problem [47]. The internal inhibitor problem will not be addressed here. The model by Lenski and Hattingh can be used to study the situation where the growth-inhibiting substance is a bacteriostatic antibiotic, one species is susceptible pathogenic bacteria, and the other is resistant pathogenic bacteria.

While the inhibitor in the model by Lenski and Hattingh lessens the reproductive rate of the susceptible organism, $\mathrm{Hsu}, \mathrm{Li}$, and Waltman present a chemostat model in [47] with a slightly different type of inhibitor. In this model, two organisms compete for nutrient in the presence of an inhibitor which is lethal to the susceptible organism (rather than merely limiting its reproductive rate) but is taken up by the resistant organism with no damaging effect. A bactericidal antibiotic is representative of this type of inhibitor. We denote the nutrient as $S$, the susceptible organism as $x$, the resistant organism as $y$, and the inhibitor as $P$; the model for this type of chemostat follows (adapted from [47]).

$$
\begin{aligned}
& \frac{d S}{d t}=\frac{F}{V} S_{o}-\frac{F}{V} S-\frac{1}{\gamma_{1}} \frac{m_{1} S}{a_{1}+S} x-\frac{1}{\gamma_{2}} \frac{m_{2} S}{a_{2}+S} y \\
& \frac{d x}{d t}=\frac{m_{1} S}{a_{1}+S} x-\frac{F}{V} x-\xi P x \\
& \frac{d y}{d t}=\frac{m_{2} S}{a_{2}+S} y-\frac{F}{V} y \\
& \frac{d P}{d t}=\frac{F}{V} P_{o}-\frac{F}{V} P-\frac{\delta P}{K+P} y \\
& S(0), P(0) \geq 0 ; x(0), y(0)>0 .
\end{aligned}
$$


In the above equations, $m_{1}, a_{1}, \gamma_{1}$ correspond to $x$, and $m_{2}, a_{2}, \gamma_{2}$ correspond to $y$, and are analogous to definitions of $m, a$, and $\gamma$ in equations (3.1) and (3.2) above, as are $F$ and $V . S_{o}$ and $P_{o}$ are the input concentrations of nutrient and inhibitor, respectively. $\delta$ is the uptake of inhibitor by $y, K$ is a half-saturation parameter for inhibitor uptake, and $\xi$ is a constant of proportionality representing the rate at which $P$ kills $x$. Since $y$ actually removes $P$ from the environment, it is a detoxifying organism.

The chemostat has been suggested for use as a model in clinical applications other than bacteria and antibiotics. For example, Edelstein-Keshet [5] outlines a simple system of two equations that can be used to describe the course of chemotherapy in a patient who is receiving drugs via an internal infusion pump. If, say, the patient has a tumor in the liver, then Edelstein-Keshet views the infusion pump as analogous to the feed bottle of the chemostat and the liver as a type of culture chamber. Tumor cells and drug units are mixed in the liver. She suggests the following as an outline for the system of equations:

$$
\begin{aligned}
& \frac{d N}{d t}=(\text { growth rate of cells })-(\text { drug induced death rate }) \\
& \left.\frac{d c}{d t}=\text { (rate drug infused }\right)-(\text { rate of cell uptake }) \text { (rate of removal by circulation) }
\end{aligned}
$$

where $N$ is the number of tumor cells per unit blood volume and $c$ is the number of units in circulation per unit blood volume.

\subsection{The One Bug-Two Drug Model of the ICU}

The ICU is similar to the chemostat with inhibitor described by $\mathrm{Hsu}, \mathrm{Li}$, and Waltman in several respects, which justifies its use as a basis for the model. However, there are some key differences which are reflected in the equations of the ICU model. The ICU itself may be viewed as the culture vessel, or bacterial growth chamber, of a chemostat. The patient admissions and discharges represent the inflow and outflow. If we assume that the ICU is always operating at capacity, then the volume is constant; i.e., inflow equals outflow, as in the chemostat. The patient hosts provide nutrient for the bacteria. There are two inhibitors in the ICU, namely, the antibiotics being administered to the patients, although the two different antibiotics are not present simultaneously. The 
inhibitor is input from an external source, so this may be classified as an external inhibitor problem.

In the six differential equations that make up the One Bug-Two Drug model, $A_{l}$ and $A_{2}$ represent the two antibiotics being cycled in the ICU, while $B_{s s}$ represents the subpopulation of the total bacterial population which is susceptible to both $A_{l}$ and $A_{2}, B_{r s}$ is the subpopulation resistant to $A_{1}$ but susceptible to $A_{2}, B_{s r}$ is the subpopulation susceptible to $A_{1}$ but resistant to $A_{2}$, and $B_{r r}$ is the subpopulation resistant to both $A_{1}$ and $A_{2}$. Before the equations are given, we briefly discuss the differences in the chemostat model and ICU model which lead to the differences seen between equations (3.3)-(3.6) and the equations below.

\section{ICU Model Assumptions:}

1. In the chemostat model, microorganisms increase in number solely by reproduction. In the ICU, bacteria not only reproduce, but also enter the ICU via patient inflow.

2. If a patient is infected with a strain of bacteria resistant to a particular antibiotic, then the patient is not treated with that antibiotic. As a result, the equations for the rates of change of $A_{1}$ and $A_{2}$ do not contain terms reflecting "uptake" by the resistant organism (see equation (3.6)).

3. In the chemostat, the inhibitor may exit the culture vessel via outflow. In the ICU, the antibiotics are removed via patient excretion.

4. Growth of bacteria in the ICU is not limited by the amount of nutrient available as it is in the chemostat. We assume that in the ICU, resource is superabundant. Thus, the growth rate for the bacteria in the ICU model does not incorporate the amount of nutrient present. Bacterial growth in the ICU is assumed to be logistic $[5,45]$.

5. Bacteria may gain resistance to an antibiotic while that antibiotic is in use (a process called "mutation"), and lose resistance when the antibiotic is no longer in use ("reverse mutation"). The equations for the rates of change of the four bacterial subpopulations contain terms accounting for the ability of the bacteria to switch from one subpopulation to another, with some restrictions (see Assumption 6, below). 
6. For our purposes, a full cycle is defined as 240 days, where $A_{l}$ is in use for the first 120 days and $A_{2}$ is in use for the second 120 days. During one-half of a cycle, bacteria may undergo one mutation, one reverse mutation, or neither of the two. Furthermore, the type of mutation or reverse mutation which may occur depends on the antibiotic in use. Bacteria may gain resistance to a particular antibiotic only if that antibiotic is in use, and lose resistance only if it is not in use. Figure 1 at right shows the possible mutations/reverse mutations. Note that this assumption
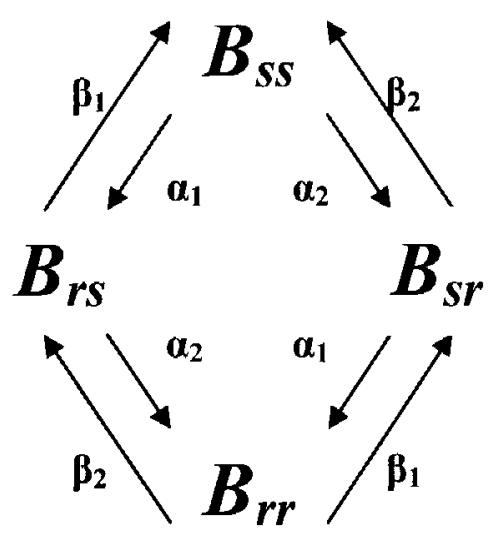

Figure 1 Pathways of Bacterial Mutation and Reverse Mutation places restrictions on the ability of bacteria to move from one subpopulation to another. For example, doubly susceptible bacteria require a full cycle to become doubly resistant, as two mutations must occur, one during each half cycle.

7. Rather than one inhibitor being present at all times as in the chemostat, in the ICU, $A_{l}$ is in use if and only if $A_{2}$ is not in use. To incorporate this effect into the model, an idealized unitless drug indicator function, $d(t)$, is defined. The drug indicator function is defined as one if $A_{1}$ is in use and zero if $A_{2}$ is in use (see Table 5). The function is time-dependent. This function will be made continuous before solving the system (see Section 4.2).

8. The antibiotic is not input at a constant rate as is the inhibitor in the chemostat model. Rather, the concentration of antibiotic depends on exactly two factors: whether the drug is in use at that time, and the number of bacteria which are susceptible to that antibiotic at any given time, up to some limiting value (i.e., amount of antibiotic present in the ICU reaches a maximum value when that antibiotic is in use and every patient is infected with susceptible bacteria). Thus the rate of increase of antibiotic displays Michaelis-Menten kinetics. The half-saturation parameter in this rate is the concentration of bacteria in the ICU at which the concentration of antibiotic is one-half of the maximum. 
The One Bug-Two Drug model follows, with parameters defined in Table 5.

$$
\begin{aligned}
& \frac{d B_{s s}}{d t}= r_{s s} B_{s s}\left(1-\frac{B_{\text {total }}}{K}\right)+\frac{F_{\text {in }}}{V} \hat{B}_{s s}-\frac{F_{\text {out }}}{V} B_{s s}-\alpha_{1} d(t) B_{s s}-\alpha_{2}(1-d(t)) B_{s s} \\
&+\beta_{1}(1-d(t)) B_{r s}+\beta_{2} d(t) B_{s r}-\xi_{1} d(t) B_{s s}-\xi_{2}(1-d(t)) B_{s s} \\
& \frac{d B_{r s}}{d t}= r_{r s} B_{r s}\left(1-\frac{B_{\text {stotal }}}{K}\right)+\frac{F_{i n}}{V} \hat{B}_{r s}-\frac{F_{\text {out }}}{V} B_{r s}+\alpha_{1} d(t) B_{s s}-\alpha_{2}(1-d(t)) B_{r s} \\
&-\beta_{1}(1-d(t)) B_{r s}+\beta_{2} d(t) B_{r r}-\xi_{2}(1-d(t)) B_{r s} \\
& \frac{d B_{s r}}{d t}= r_{s r} B_{s r}\left(1-\frac{B_{t o t a l}}{K}\right)+\frac{F_{i n}}{V} \hat{B}_{s r}-\frac{F_{\text {out }}}{V} B_{s r}-\alpha_{1} d(t) B_{s r}+\alpha_{2}(1-d(t)) B_{s s} \\
&+\beta_{1}(1-d(t)) B_{r r}-\beta_{2} d(t) B_{s r}-\xi_{1} d(t) B_{s r} \\
& \frac{d B_{r r}}{d t}= r_{r r} B_{r r}\left(1-\frac{B_{t o t a l}}{K}\right)+\frac{F_{i n}}{V} \hat{B}_{r r}-\frac{F_{\text {out }}}{V} B_{r r}+\alpha_{1} d(t) B_{s r}+\alpha_{2}(1-d(t)) B_{r s} \\
&-\beta_{1}(1-d(t)) B_{r r}-\beta_{2} d(t) B_{r r} \\
& \frac{d A_{1}}{d t}= \frac{\rho_{1}\left(B_{s s}+B_{s r}\right)}{\kappa_{1}+B_{s s}+B_{s r}} d(t)-\gamma_{1} A_{1} \\
& \frac{d A_{2}}{d t}= \frac{\rho_{2}\left(B_{s s}+B_{r s}\right)}{\kappa_{2}+B_{s s}+B_{r s}}(1-d(t))-\gamma_{2} A_{2} \\
& B_{s s}(0), B_{r s}(0), B_{s r}(0), B_{r r}(0) \geq 0 ; A_{1}(0), A_{2}(0) \geq 0 \\
&\left(B_{i j}(0)>0\right. \text { for some subpopulation). }
\end{aligned}
$$

Before we proceed to the analysis of the model, we address two valid criticisms of the chemostat as a model for the ICU. The first is that in the chemostat model, it is assumed that the bacterial growth chamber is kept well stirred. In the clinical setting, obviously it is not desirable that the contents of the ICU be "well stirred." In fact, while cross-contamination does occasionally occur, resulting in the nosocomial infections discussed in Chapter II, hospital personnel generally go to great lengths to prevent this. The second criticism is that the One Bug-Two Drug model contains no equation reflecting rate of change of nutrient. This difference is not significant, because "nutrient" is assumed to be superabundant. Note that Edelstein-Keshet's system of equations describing the interaction of drugs and tumor cells in the liver contains no nutrient equation. In addition to these considerations, the conditions in the ICU merit a 
Table 5 Parameters in the One Bug-Two Drug Model

\begin{tabular}{|c|c|c|}
\hline Parameter & Units & Description \\
\hline $\begin{array}{c}B_{s s}, B_{r s}, B_{s r}, B_{r r} \\
B_{\text {total }}\end{array}$ & $\mathrm{CFU} / \mathrm{mL}$ & $\begin{array}{l}\text { Concentrations of each of the four types of bacteria } \\
\text { in the ICU; } B_{\text {total }} \text { is the sum of } B_{s s}, B_{r s}, B_{s r} \text {, and } B_{r r} \text {. }\end{array}$ \\
\hline$A_{1}, A_{2}$ & $\mu \mathrm{g} / \mathrm{mL}$ & Concentrations of each of antibiotics 1 and 2 in the ICU \\
\hline$K$ & $\mathrm{CFU} / \mathrm{mL}$ & Carrying capacity of the total bacterial population \\
\hline$V$ & $\mathrm{~mL}$ & Volume of ICU \\
\hline$\hat{B}_{s s}, \hat{B}_{r s}, \hat{B}_{s r}, \hat{B}_{r r}$ & $\mathrm{CFU} / \mathrm{mL}$ & $\begin{array}{l}\text { Concentrations of each of the four types of bacteria } \\
\text { in a typical incoming ICU patient }\end{array}$ \\
\hline $\begin{array}{l}r_{s s}, r_{r s}, r_{s r}, r_{r r} \\
F_{\text {in }}, F_{\text {out }}\end{array}$ & $\begin{array}{l}1 / \text { time } \\
\mathrm{mL} / \text { time }\end{array}$ & $\begin{array}{l}\text { Growth rates of each of the four types of bacteria } \\
\text { Volumetric patient inflow/outflow rates }\end{array}$ \\
\hline$\alpha_{1}, \alpha_{2}$ & $1 /$ time & $\begin{array}{l}\text { Mutation rates; rates at which bacteria gain resistance } \\
\text { to } A_{l} \text { and } A_{2} \text {, respectively }\end{array}$ \\
\hline$\beta_{1}, \beta_{2}$ & $1 /$ time & $\begin{array}{l}\text { Reverse mutation rates; rates at which bacteria } \\
\text { lose resistance to } A_{I} \text { and } A_{2} \text {, respectively }\end{array}$ \\
\hline$\xi_{1}, \check{\zeta}_{2}$ & $1 /$ time & $\begin{array}{l}\text { Killing rates; rates at which susceptible bacteria are } \\
\text { killed by } A_{l} \text { and } A_{2} \text {, respectively }\end{array}$ \\
\hline$\rho_{l}, \rho_{2}$ & $\mu \mathrm{g} /(\mathrm{mL}-\mathrm{time})$ & Maximum concentrations of $A_{1}$ and $A_{2}$, respectively \\
\hline$\kappa_{1}, \kappa_{2}$ & $\mathrm{CFU} / \mathrm{mL}$ & $\begin{array}{l}\text { Michaelis-Menten constant; concentration of } \\
\text { bacteria susceptible to } A_{l}, A_{2} \text { at which dosage of } \\
A_{l}, A_{2} \text { is one-half of the maximum, respectively }\end{array}$ \\
\hline$\gamma_{1}, \gamma_{2}$ & 1/time & Elimination rate constants for $A_{l}$ and $A_{2}$, respectively \\
\hline$d(t)$ & none & Drug indicator function: \\
\hline (idealized) & & $\begin{array}{l}1 \text { for } 0<t \leq 120,240<t \leq 360 \\
0 \text { for } 120<t \leq 240,360<t \leq 480\end{array}$ \\
\hline
\end{tabular}

slightly different view of the chemostat which render a nutrient equation unnecessary, as explained below. 
Let us return to the model of the simple chemostat, where no inhibitor is present (equations (3.1) and (3.2)). The rate of change of antibiotic in the ICU is analogous to the rate of change of organism in the simple chemostat in several ways. The similarities are listed in Table 6. (The chemostat assumptions are given by Edelstein-Keshet in [5].) Essentially, if one takes this point of view, the ICU is no longer viewed as a chemostat with inhibitor. Instead, it is viewed as a simple chemostat. The antibiotics in the ICU correspond to the organisms in the culture vessel of the chemostat, and the bacteria in the ICU are analogous to "nutrient," the substance whose availability (or lack thereof) causes the amount of antibiotic to increase or decrease. While this is a somewhat unconventional view of the chemostat, it closely follows Edelstein-Keshet's vision of the chemostat as a model for chemotherapy and it accurately describes the interplay between bacteria and antibiotics in the ICU in several ways.

Table 6 Comparison of Simple Chemostat and ICU

Chemostat

The growth rate of microorganism depends on nutrient availability.

Nutrient depletion occurs continuously as a result of reproduction by microorganism.

Rate of growth of microorganism increases with nutrient availability only up to some limiting value; microorganism growth rate and nutrient consumption is assumed to be a saturating function of nutrient concentration.

Nutrient is continually added to the culture vessel via inflow.

\section{ICU}

The increase or decrease in antibiotic concentration depends on number of susceptible bacteria present in the ICU.

Susceptible bacteria decrease in number continuously as a result of increase in antibiotic concentration.

Rate of "growth" of antibiotic concentration increases depending on number of susceptible bacteria only up to some limiting value; antibiotic concentration and bacterial killing is assumed to be a saturating function of bacterial concentration.

Susceptible bacteria continually enter the ICU via patient admission. 


\title{
CHAPTER IV
}

\section{ANALYSIS OF MODEL}

\author{
"The exploration of the conditions which do, and which do not, influence a \\ phenomenon is part of the early experimental exploration of a field. "
}

Eugene P. Wigner, The Unreasonable Effectiveness of Mathematics in the Natural Sciences

\subsection{The LHS and LHS/PRC Analysis Techniques}

It is often the case in modeling biological phenomena that there is a significant amount of uncertainty as to the true values of the inputs that will be used to evaluate the model outcome. In some cases, an input value may not be constant, but may vary within a known range of values. In other cases, it may be impossible to estimate the input value (or even range of values) to the desired degree of accuracy. For example, Blower and Dowlatabadi [9] present a model of HIV transmission in which one of the parameters is the rate of needle sharing among IV drug users; certainly it is difficult to pinpoint an accurate value for such a parameter.

The purpose of uncertainty analysis is to evaluate the variability of the model outcome due to changes in the input values. The behavior of a deterministic model such as the One Bug-Two Drug model is determined solely by its time evolution equations and input values (initial conditions and parameter values), so uncertainty analysis will produce quantitative information about the imprecision of the outcome of a deterministic model. Sensitivity analysis extends uncertainty analysis by identifying the input values which have the greatest effect on model results. Sensitivity analysis is instrumental in both interpreting model results and guiding future research efforts [51]. 
The LHS (Latin Hypercube Sampling) uncertainty and LHS/PRC (Latin Hypercube Sampling/Partial Rank Correlation) sensitivity analysis techniques were used to analyze the One Bug-Two Drug model in this paper. The LHS technique was proposed by McKay, Conover, and Beckman in [52]. They compared three methods of selecting, or "sampling," input values: random sampling, stratified sampling, and Latin hypercube sampling. Their results proved that unbiased estimates of the sample mean could be obtained with the LHS design, and that the estimator of the mean using the LHS design was clearly superior to both the random sampling and stratified sampling designs when using the same number of model simulations for each method. The LHS design has also been proven to be an extremely efficient design. Seaholm, et al. [53] compared the LHS technique with a full factorial (FF) fixed point sample and found that an FF design with over 14 times as many samples as the LHS design produced approximately the same predictive ability and information about model uncertainty. While LHS has its advantages over other methods, one drawback is that it does not evaluate the effects of individual input values on the model outcome [54]. For this reason, partial rank correlation coefficients are often used in combination with LHS $[9,54,55]$. The use of the partial rank correlation coefficient (PRCC) is not uncommon in sensitivity studies [51], but it is not informative in cases where there exists a nonmonotonic relationship between variables. Such relationships must be investigated by other means.

As outlined in [9], there are seven steps in the LHS and LHS/PRC techniques:

1. Suppose that the model has $\mathrm{k}$ uncertain input values. Assign a probability distribution function (pdf) to each of these $k$ input variables. In the case of a deterministic model, this means that all parameters and initial conditions which are not known constants are assigned probability distribution functions. These pdf's should be obtained from a search of relevant literature, experimental data, or other methods whenever possible. O'Neill, et al. recommend that a parameter be assigned the triangular distribution when the true distribution of the parameter is unknown, but a minimum, maximum, and most likely value for the parameter are known [56].

2. Determine the number of simulations, $N$. The inequalities $N>4 k / 3$ and $\mathrm{N} \geq(\mathrm{k}+1)$ must be satisfied [9], although there is no formula to determine an optimal value for $\mathrm{N}$. 
3. Divide the range of each of the $\mathrm{k}$ parameters into $\mathrm{N}$ strata of equal probability. (The area under the curve of each interval is now 1/N.) For each of the k parameters, select exactly one value at random from each of the $\mathrm{N}$ intervals. For example, if $\mathrm{x}$ is a parameter, then the set $\left\{\mathrm{x}_{1}, \mathrm{x}_{2}, \ldots, \mathrm{x}_{\mathrm{N}}\right\}$ represents the $\mathrm{N}$ values selected for $\mathrm{x}$, where $\mathrm{x}_{1}$ is selected from the first equiprobable interval, $x_{2}$ is selected from the second equiprobable interval, and so on. Note that in this step of the LHS scheme, the entire range of each parameter is represented. Thus sampling occurs over all of the sample space, which is one of the advantages of the LHS technique.

4. Create the LHS table. The LHS table is an N $\times k$ matrix, where column $j(j=1$,

$2, \ldots, \mathrm{k}$ ) is a random permutation of the $\mathrm{N}$ values selected for the $\mathrm{j}^{\text {th }}$ parameter in step 3 , above. Each row of the LHS table is a k-tuple which will be used as an input vector for exactly one simulation of the model.

5. Perform the $\mathrm{N}$ simulations of the model. Row $\mathrm{i}(\mathrm{i}=1,2, \ldots, \mathrm{N})$ of the LHS table is the input vector for the $\mathrm{i}^{\text {th }}$ run of the model.

6. Perform uncertainty analysis.

7. Calculate partial rank correlation coefficients (PRCC's) between each parameter and each outcome variable; perform sensitivity analysis.

\subsection{Uncertainty Analysis}

We now proceed to the implementation of the LHS uncertainty analysis procedure for the One Bug-Two Drug model. First, probability distribution functions must be assigned to each parameter. A search of the medical literature did not produce any specified pdf's for the input variables; however, in most cases a minimum value and maximum value could be ascertained from the literature. The minimum and maximum values for the initial conditions and some of the parameters were established through personal communication with Dr. D.T. Tsukayama, an infectious disease specialist at Hennepin County Medical Center in Minneapolis, Minnesota. All pdf's are assumed to be triangular, with the most likely value (peak) at the midpoint between the minimum and maximum values. The pdf's are defined in Table 7 , accompanied by a discussion of the biological justifications for the definitions. 
We assume that initial conditions are zero for both $A_{1}$ and $A_{2}$, as the appropriate amount of antibiotic will be determined according to whether the antibiotic is in use and susceptible bacteria are present in the ICU. The minimum and maximum values of the total initial bacterial concentration were established by assuming that 6-12 patients are infected with bacteria at time $\mathrm{t}=0$, and of these bacteria, $80 \%$ are doubly susceptible, $9 \%$ are singly resistant to either $A_{I}$ or $A_{2}$, and $2 \%$ are doubly resistant. Thus initial conditions for concentrations of bacterial subpopulations are

$$
\begin{aligned}
& B_{s s}(0)=0.8 \times B_{\text {total }}(0), \\
& B_{r s}(0)=B_{s r}(0)=0.09 \times B_{\text {totat }}(0), \text { and } \\
& B_{r r}(0)=0.02 \times B_{\text {totat }}(0),
\end{aligned}
$$

where $B_{\text {total }}(0)$ is total initial bacterial concentration. The minimum count per site necessary for infection to occur was assessed by Dr. Tsukayama. Minimum and maximum values of $\hat{B}_{s s}, \hat{B}_{r s}, \hat{B}_{s r}, \hat{B}_{r r}$ were calculated in a similar fashion. A plausible range for the maximum carriage of bacteria in host tissue was established, and the minimum and maximum values of the carrying capacity $(K)$ were found by assuming that this concentration of bacteria was present in all of the 12 ICU patients. The range of the growth rate of doubly susceptible bacteria, $r_{s s}$, was found in [57]. A search of the literature provided no information about the growth rates $r_{r s}, r_{s r}$, and $r_{r r}$. However, it has been established that gaining resistance imposes a fitness cost on the bacteria [43]. For the purpose of this analysis, it was assumed that the growth rate $r_{s s}$ decreases by $20 \%$ with a single resistance-conferring mutation and by $40 \%$ with two such mutations; maximum and minimum values for the three remaining growth rates were calculated based on this assumption.

The range of values for patient inflow rate, $F_{i n}$, was based on the assumption that 3-5 patients are admitted to the ICU each day; furthermore, the ICU in this case is kept at capacity, so $F_{\text {out }}$ and $F_{\text {in }}$ are equal. Because of this assumption of equality, $F_{\text {in }}$ and $F_{\text {out }}$ will both be referred to as simply $F$, the patient inflow/outflow rate, in the analysis of the model. The mutation rate for ciprofloxacin, $a_{l}$, was found in [58]. While an exact range of values for the mutation rate for piperacillin/tazobactam could not be found, it is certain that the resistance potential for ciprofloxacin is high but the resistance potential for 
Table 7 Probability Distribution Functions for Input Variables

\begin{tabular}{|c|c|c|c|c|c|}
\hline & Min. & Max. & & Min. & Max. \\
\hline Initial & & & Parameters & & \\
\hline Condition & & & (continued) & & \\
\hline$B_{\text {total }}(0)$ & $5.0 \times 10^{3}$ & $1.0 \times 10^{7}$ & $\alpha_{2}$ & $1.5625 \times 10^{-7}$ & $3.125 \times 10^{-6}$ \\
\hline Parameters & & & $B_{l}$ & $7.8125 \times 10^{-8}$ & $1.5625 \times 10^{-6}$ \\
\hline$K$ & $1.0 \times 10^{12}$ & $1.0 \times 10^{15}$ & $B_{2}$ & $3.90625 \times 10^{-8}$ & $7.8125 \times 10^{-7}$ \\
\hline$\hat{B}_{\text {total }}$ & $1.0 \times 10^{4}$ & $1.0 \times 10^{7}$ & $\xi_{I}$ & 0.96 & 0.99 \\
\hline$r_{s s}$ & 36.97 & 45.36 & $\xi_{2}$ & 0.96 & 0.99 \\
\hline$r_{r s}$ & 29.576 & 36.288 & $\kappa_{l}$ & $4.45 \times 10^{3}$ & $4.45 \times 10^{6}$ \\
\hline$r_{s r}$ & 29.576 & 36.288 & $\kappa_{2}$ & $4.45 \times 10^{3}$ & $4.45 \times 10^{6}$ \\
\hline$r_{r r}$ & 22.182 & 27.216 & $\gamma_{l}$ & 1.92 & 7.68 \\
\hline$F$ & $5.82 \times 10^{5}$ & $1.15 \times 10^{6}$ & $\gamma_{2}$ & 12.96 & 19.2 \\
\hline$\alpha_{l}$ & $3.125 \times 10^{-7}$ & $6.25 \times 10^{-6}$ & & & \\
\hline
\end{tabular}

piperacillin/tazobactam is low $[22,27,28,29,59]$. For the purpose of model analysis, we assume that the minimum and maximum values for $\alpha_{2}$ are approximately one-half of the minimum and maximum values for $\alpha_{l}$. Reverse mutation rates rarely, if ever, appear in the literature; in this case no reverse mutation rates could be found. Nevertheless, loss of resistance generally occurs more slowly than development of resistance [31]. Here we assume that reverse mutation takes longer than mutation by approximately a factor of four, so maximum and minimum values for $B_{1}$ and $B_{2}$ were determined using this assumption. The killing rates, $\xi_{1}$ and $\xi_{2}$, were found in [60]. The parameters $\rho_{l}$ and $\rho_{2}$ are constants. The maximum amount of antibiotic is present when the ICU is at capacity and every patient is being dosed daily, so $\rho_{1}$ and $\rho_{2}$ are determined by multiplying the amount of antibiotic in a single daily dose by the maximum number of patients in the ICU. Here

$$
\begin{aligned}
& \rho_{1}=12 \times 800=9600 \mu \mathrm{g} /(\mathrm{mL}-\text { time }) \text { and } \\
& \rho_{2}=12 \times 13,500=162,000 \mu \mathrm{g} /(\mathrm{mL}-\text { time })[36] .
\end{aligned}
$$

The minimum and maximum values for the half-saturation parameters $\kappa_{l}$ and $\kappa_{2}$ were found by calculating the range of bacteria present in the ICU when one-half of the patients are infected with susceptible bacteria. Ranges for elimination rate constants $\gamma_{l}$ 
and $\gamma_{2}$ were found in $[61,62,63]$ and in consultation with Dr. Tsukayama. Finally, the volume of the ICU, $V$, is a constant, and was defined for this analysis as the sum of the volumes of the patients, i.e., $V$ is $2.52 \times 10^{6} \mathrm{~mL}$.

Several of the input variables will not be assigned pdf's' (the six initial conditions, $\hat{B}_{s s}, \hat{B}_{r s}, \hat{B}_{s r}, \hat{B}_{r r}, F_{\text {out }}, \rho_{l}, \rho_{2}, V$ for the reasons discussed above, although pdf's will be assigned to $B_{\text {tota }}(0)$ and $\hat{B}_{\text {total }}$. As a result, the number of input parameters to be sampled is 18 . The number of simulations is set at 200 , a number which satisfies both inequalities given in step 2 of Section 4.1. For each of the 200 simulations, we assume that ciprofloxacin was the drug in use for the first and third 120-day periods, and piperacillin/tazobactam was the drug in use during the second and fourth 120-day periods. The outcome variables are evaluated at time $t=480$ days, at the completion of two full cycles.

All of the relevant calculations in steps 3 through 7 of the LHS uncertainty and LHS/PRC sensitivity analysis (division of parameter ranges into equiprobable intervals, permutations of sampled values, evaluation of outcome variables for each of the 200 simulations, etc.) were performed with the aid of the Mathematica system. The computer program that was used to perform the model analysis was developed specifically for this paper, with [64] serving as a valuable resource in the writing of the program. In order to run the model simulations with Mathematica, the drug indicator function was redefined. While the definition of the drug indicator function in Table 5 represents an idealized vision of the cycling strategy, redefining the function as a continuous one is a better representation of reality and aids computing capability in obtaining a numerical approximation for the solution of the system of differential equations. The redefined drug indicator function incorporates a 24 hour period at days 120,240, and 360 during which usage of one drug decreases to zero and usage of the other drug increases to the appropriate level. The computer code which comprises this program, including the redefined drug indicator function, can be found in Appendix 1.

The uncertainty analysis for the antibiotic outcome variables (see equations (3.11) and (3.12)) was not particularly informative. At time $\mathrm{t}=480, A_{l}$ has been out of use for 120 days, so the concentration of this antibiotic in the ICU should be very low. The uncertainty analysis reveals this to be true, as the mean and variance of the outcome 
values of $A_{1}$ are both approximately zero. The mean of the outcome values of $A_{2}$ is $10,139 \mu \mathrm{g} / \mathrm{mL}$. This is a somewhat low concentration of antibiotic, implying a low concentration of bacteria susceptible to $A_{2}$. The results of the uncertainty analysis for the outcome variables related to bacterial density, (equations (3.7)-(3.10)), are shown in Table 8 and Figure 2, with interpretation of results given in Chapter 5. The LHS uncertainty analysis showed that the variances of the outcome variables are fairly high, indicating a high degree of model imprecision due to imprecise parameter estimation.

Table 8 Descriptive Statistics for Outcome Variables at Time $t=480$ days

\begin{tabular}{lcccc}
\hline & $B_{s s}$ & $B_{r s}$ & $B_{s r}$ & $B_{r r}$ \\
\hline Minimum & $4.14099 \times 10^{13}$ & $12,603.1$ & $1.18013 \times 10^{9}$ & $61,245.4$ \\
Maximum & $9.85409 \times 10^{14}$ & 291,951 & $6.38529 \times 10^{10}$ & $1.92631 \times 10^{11}$ \\
Mean & $4.96502 \times 10^{14}$ & 147,168 & $1.33536 \times 10^{10}$ & $1.78357 \times 10^{9}$ \\
Variance & $4.12099 \times 10^{28}$ & $3.74766 \times 10^{9}$ & $9.33395 \times 10^{19}$ & $2.22959 \times 10^{20}$ \\
\hline
\end{tabular}

(a)

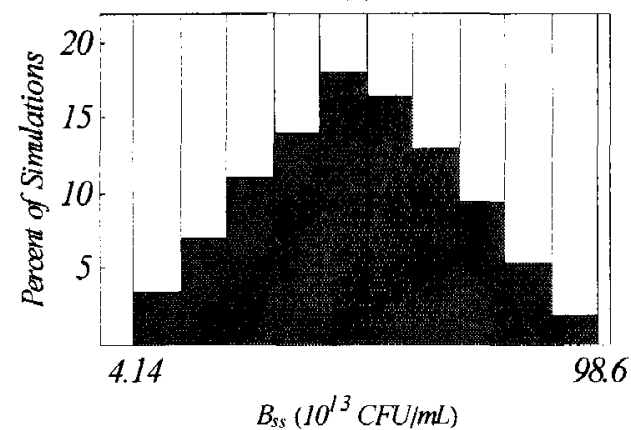

(c)

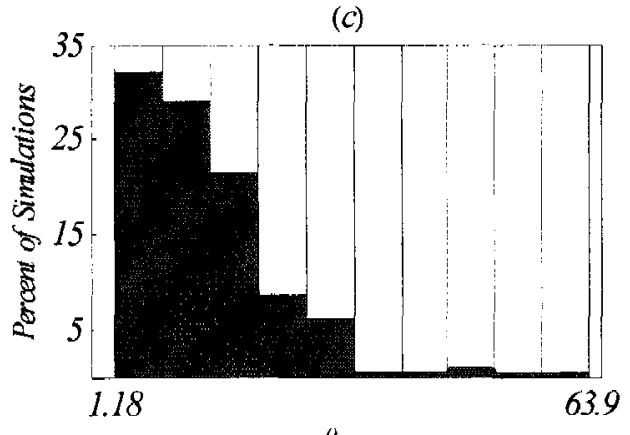

$B_{s r}\left(10^{9} \mathrm{CFU} / m L\right)$

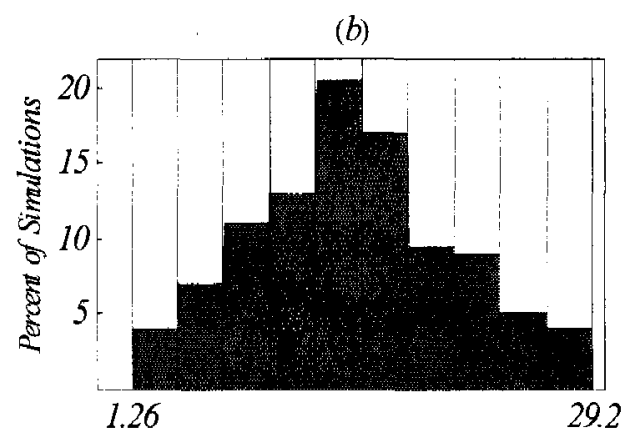

$B_{r s}\left(l O^{+} \mathrm{CFU} / \mathrm{mL}\right)$

(d)

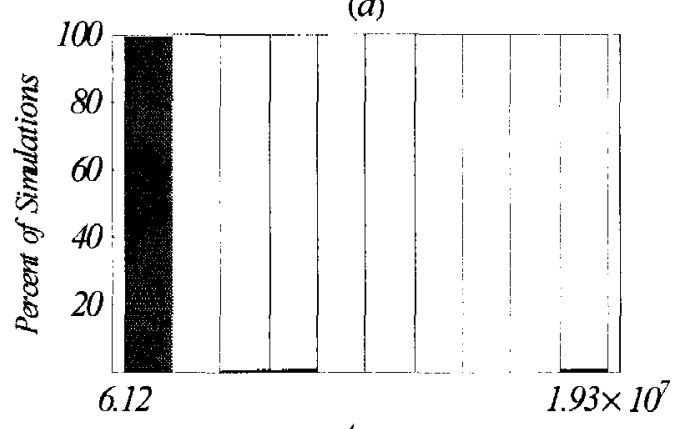

$B_{r r}\left(I O^{+} C F U / m L\right)$

Figure 2 Frequency Distributions for Values of Outcome Variables (a) $B_{s s}$, (b) $B_{r s}$, (c) $B_{s r}$, and (d) $B_{r r}$ at time $\mathrm{t}=480$ days. 


\subsection{Sensitivity Analysis}

Partial rank correlation coefficients between each sampled input variable and each outcome variable were calculated using formulae from $[9,65,66]$ and the Mathematica program. A complete description of the methods by which PRCC's were calculated is given in Appendix 2. A PRCC may range in value from -1 to 1 . The greater the magnitude of the PRCC between a parameter and an output variable, the more impact the parameter has on the outcome of the variable. A positive value of the PRCC indicates that as the input value increases, the output value increases. A PRCC with a negative sign denotes an inverse relationship between the input and output values. The PRCC's for the antibiotic outcome variables are not of interest here, as few parameters affect the outcome of antibiotic concentration. For each of the four outcome variables related to bacterial density, the PRCC's with significance levels 0.001 and 0.05 were identified. Results are shown in Table 9 and discussed in Chapter 5.

Table 9 Partial Rank Correlation Coefficients

\begin{tabular}{|c|c|c|c|}
\hline \multicolumn{2}{|c|}{$B_{s s}$} & \multicolumn{2}{|c|}{$B_{r s}$} \\
\hline parameter & PRCC & parameter & PRCC \\
\hline$K$ & $0.99^{\mp}$ & $\hat{B}_{\text {total }}$ & $0.99^{7}$ \\
\hline \multirow[t]{3}{*}{$F$} & $-0.31^{*}$ & $F$ & $0.88^{\ddagger}$ \\
\hline & & $r_{r s}$ & $0.18^{\dagger}$ \\
\hline & & $r_{s s}$ & $-0.16^{\dagger}$ \\
\hline \multicolumn{2}{|c|}{$B_{s r}$} & \multicolumn{2}{|c|}{$B_{r r}$} \\
\hline parameter & PRCC & parameter & PRCC \\
\hline$K$ & $0.87^{7}$ & $F$ & $-0.69^{\mp}$ \\
\hline$\alpha_{2}$ & $0.86^{\ddagger}$ & $r_{s s}$ & $-0.42^{\star}$ \\
\hline$r_{s s}$ & $-0.64^{\ddagger}$ & $r_{r r}$ & $0.41^{*}$ \\
\hline$r_{s r}$ & $0.63^{\ddagger}$ & $\hat{B}_{t, t a l}$ & $0.17^{\dagger}$ \\
\hline$F$ & $-0.52^{\ddagger}$ & & \\
\hline Results are & gnifice & the $0.05 \mathrm{le}$ & $1\left(^{\dagger}\right)$ \\
\hline or $0.001 \mathrm{le}$ & $\left({ }^{+}\right)$. & & \\
\hline
\end{tabular}




\section{CHAPTER V \\ CONCLUSIONS}

"Anything that is produced by evolution is bound to be a bit of a mess."

Sydney Brenner,

Quoted in Why is development so illogical?

In this chapter we begin with a discussion of the results of the uncertainty and sensitivity analysis of Chapter 4 , accompanied by an examination of the behavior of the model, then briefly discuss experimental data, and conclude with some suggestions that will be useful in guiding future work with the One Bug-Two Drug model.

The behavior of the model was fairly stable in each of the 200 simulations; no chaotic behavior was observed. In order to gain a clearer understanding of the model, a simulation was performed in which each input variable (both parameters and initial conditions) was assigned the value of the mean of its pdf (see Table 7). The model's four outcome variables relating to bacterial density were plotted with respect to time. The results are shown in Figure 3. In the case of the first three variables, $B_{s s}, B_{r s}$, and $B_{s r}$, the bacterial concentration quickly reaches a maximum or minimum value during each 120 day period and remains at that value until the next drug is cycled into use. The fourth variable, $B_{r r}$, behaves somewhat differently. At the beginning of each 120 day period, the concentration of $B_{r r}$ quickly rises to some peak value, and then declines to a minimum value for the remainder of the 120 day period. The peak value of $B_{r r}$ at day 240 is much lower than the peaks on days 120 and 360 and cannot be seen in Figure 3 due to the scale of the graph. The peak occurring on day 240 is shown in Figure 4. The scale of the 
(a) $B_{s s}$

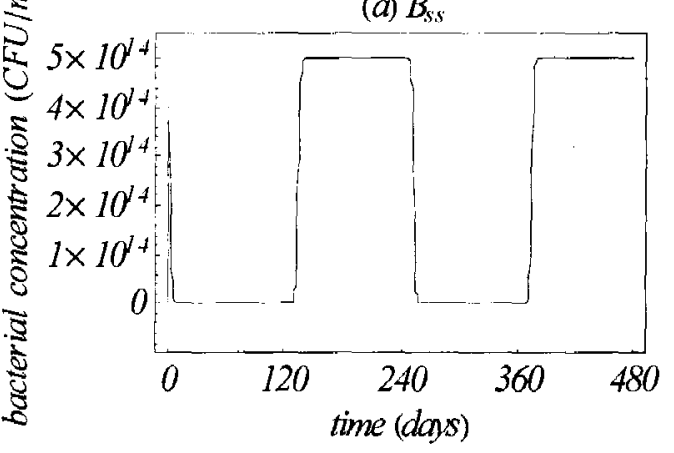

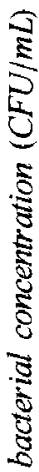

(c) $B_{s r}$

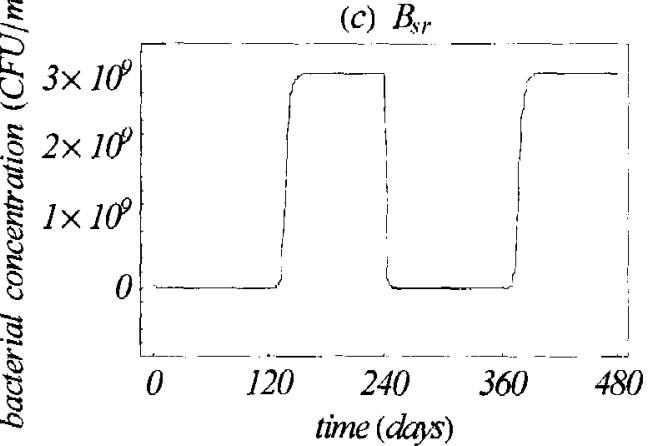

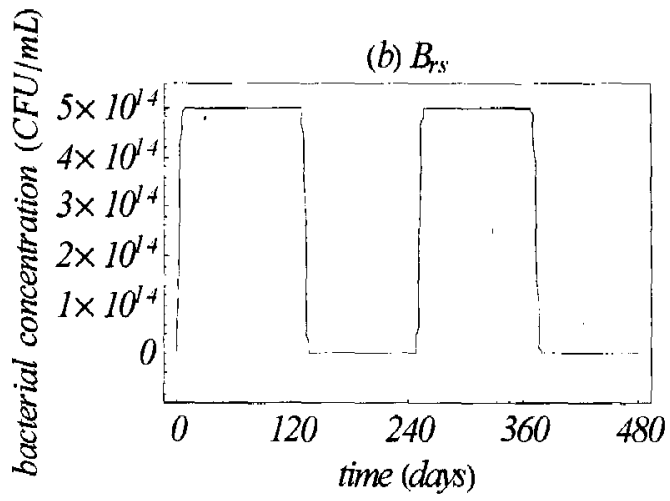

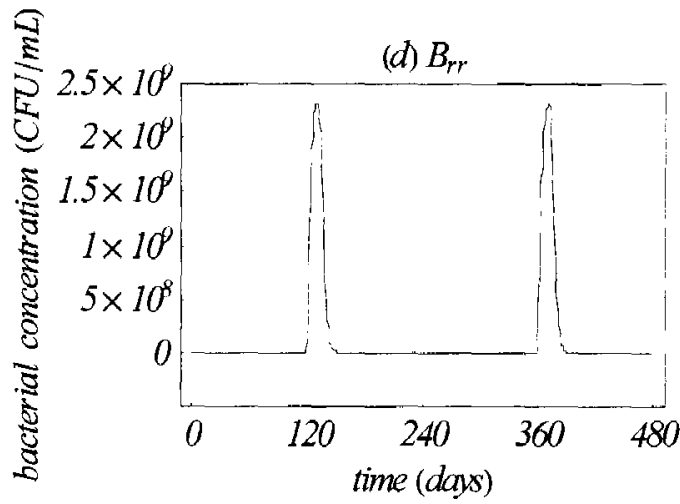

Figure 3 Model Simulation at Mean Input Values for

(a) $B_{s s}$, (b) $B_{r s}$, (c) $B_{s r}$, and (d) $B_{r r}$.

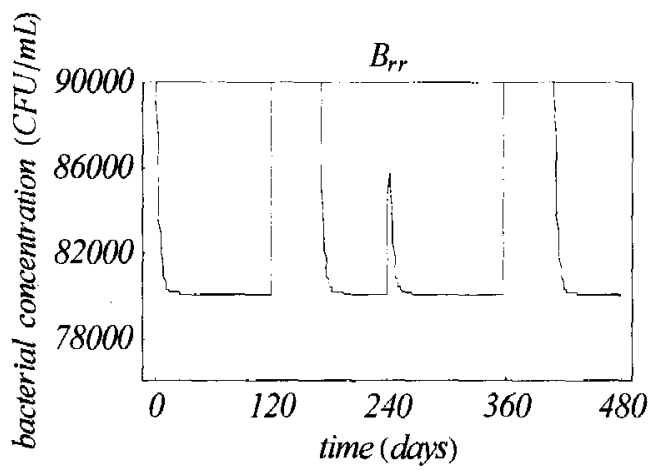

Figure 4 Peak in $B_{r r}$ Concentration at Day 240. 
graphs in Figure 3 also makes it difficult to see the minimum values of the outcome variables. Although they appear to be zero in each case, in fact, the minimum bacterial concentrations of these variables remain above zero. Minimum values for $B_{s s}, B_{r s}, B_{s r}$ and $B_{r r}$ are approximately $1.129 \times 10^{6}, 1.27 \times 10^{5}, 9.97 \times 10^{4}$, and $8 \times 10^{4} \mathrm{CFU} / \mathrm{mL}$, respectively, in the simulation at mean input values. While maximum and minimum values may vary for each of the 200 model simulations, the behavior seen in Figure 3 is roughly representative of the behavior seen in each simulation. The nonzero minimum values for the model indicate that the infections in the ICU are not clearing. This points to a model deficiency, as there will be times when no $P$. aeruginosa infections are present in the ICU in reality.

The frequency distributions of the outcome variables in Figure 2 show that the outcome values for $B_{s s}$ and $B_{r s}$ are approximately symmetric about the mean, but the outcome values for $B_{s r}$ and $B_{r r}$ are right-skewed. In the case of $B_{r r}, 197$ of the 200 model simulations produced outcome values in the lowest tenth of the interval between the minimum and maximum outcome values for $B_{r r}$. The three model simulations which yielded outcome values outside of this range are attributed to the coupling of very high input values for the growth rate $r_{r r}$ with very low values for the patient inflow/outflow rate $F$. (The implications of these input values on the outcome of $B_{r r}$ will be discussed later in this chapter.) A high concentration of $B_{r r}$ is likely to contribute to a higher concentration of $B_{s r}$, as a greater number of bacteria are losing resistance to $A_{l}$ and moving from subpopulation $B_{r r}$ to subpopulation $B_{s r}$ at time $\mathrm{t}=480$, after $A_{2}$ has been in use for 120 days.

The use of partial rank correlation coefficients in the sensitivity analysis allows us to determine which parameters are most responsible for producing the variability in the output values. Some of the results obtained in the sensitivity analysis of the One BugTwo Drug model (see Table 9) are fairly obvious, while others are more surprising. For example, the PRCC's of $r_{s s}$ with respect to the bacterial subpopulations $B_{r s}, B_{s r}$, and $B_{r r}$ are negative. This is probably due to the fitness advantage of doubly susceptible bacteria [43]; when $B_{s s}$ makes up a larger portion of the carrying capacity, there is less "room" for the other types of bacteria. Note also that the positive correlation between the carrying capacity $K$ and $B_{s s}$ is very high. At time $\mathrm{t}=480$, the concentration of $B_{s s}$ is being reduced 
by patient outflow $(F)$, bacterial killing by $A_{2}\left(\xi_{2}\right)$, and mutation $\left(\alpha_{2}\right)$. However, sensitivity analysis indicates that patient outflow has a greater effect on the outcome than either killing or mutation.

The results of the sensitivity analysis become more interesting in studying the behavior of $B_{r s}$ and $B_{s r}$. At time $\mathrm{t}=480$, the concentration of $B_{r s}$ is at a minimum, so the growth term in equation (3.8) containing the parameters $r_{r s}$ and $K$ has little effect on the outcome. The PRCC of $K$ with respect to $B_{r s}$ was not significant, and the PRCC of $r_{r s}$ with respect to $B_{r s}$ shows a relatively small positive correlation. Hence the concentration increases primarily through patient inflow, as evidenced by the strong positive correlation of both $\hat{B}_{\text {rotal }}$ and $F$ with respect to $B_{r s}$. Mutation, reverse mutation, and killing rates did not have a significant effect on the outcome of $B_{r s}$, possibly because $B_{r s}$ concentration is very low at day 480 . For the outcome variable $B_{s r}, \alpha_{2}$ is a significant parameter because $A_{2}$ has been in use for the past 120 days, so the concentration is increasing due to mutation of $B_{s s}$, a bacterial subpopulation which is at fairly high levels at day 480 . Growth rate $r_{s r}$ and carrying capacity $K$ are also clearly important, since the concentration of $B_{s r}$ is high at time $\mathbf{t}=480$. Note that the concentration of $B_{s r}$ has not been reduced through bacterial killing by $A_{2}$ because of its resistance, so we see a strong negative correlation between $F$ and $B_{s r}$, indicating that this subpopulation is being reduced primarily through patient outflow. Although $B_{s r}$ is losing resistance to $A_{l}$, reverse mutation rate $B_{l}$ does not have a significant effect.

Before discussing the sensitivity analysis results for $B_{r r}$, we note that the significance of the input variables often varies with respect to time. Table 10 shows a comparison of the PRCC's of selected parameters with respect to $B_{r s}$ and $B_{s r}$ at days 360 and 480. Consider the results of Table 10 in light of the model behavior shown in Figure 3. Here it is clearly evident that when bacterial concentration is at a maximum, growth rate and carrying capacity are more strongly correlated with bacterial outcome than when bacterial concentration is at a minimum. Note also that the significance of the parameters varies depending on the drug that is in use. For example, the effect of mutation rate $\alpha_{2}$ on the outcome value of $B_{s r}$ is insignificant at time $\mathrm{t}=360$, because $A_{l}$ has been in use for the previous 120 days. In addition, the comparison of PRCC's indicates that when 
Table 10 Comparison of Partial Rank Correlation Coefficients with respect to $B_{r s}$ and $B_{s r}$ at Times $\mathrm{t}=360$ and $\mathrm{t}=480$ days

\begin{tabular}{cccccc}
\hline$B_{r s}$ & $t=360$ & $\mathrm{t}=480$ & $B_{s r}$ & $\mathrm{t}=360$ & $\mathrm{t}=480$ \\
\hline$\hat{B}_{\text {total }}$ & -0.05 & 0.99 & $\hat{B}_{\text {toral }}$ & 0.97 & 0.09 \\
$F$ & -0.36 & 0.88 & $\alpha_{2}$ & -0.04 & 0.86 \\
$r_{r s}$ & 0.23 & 0.18 & $r_{s r}$ & 0.22 & 0.63 \\
$K$ & 0.99 & 0.11 & $F$ & 0.76 & -0.52 \\
& & & $\xi_{1}$ & -0.13 & -0.03 \\
& & & $K$ & 0.15 & 0.87 \\
\hline
\end{tabular}

bacterial concentration is low, the primary means of increase in concentration is via patient inflow.

The sensitivity analysis results at time $\mathrm{t}=480$ for $B_{r r}$ (Table 9) show that decrease in the concentration of $B_{r r}$ occurs primarily through patient outflow $(F)$. The double resistance of $B_{r r}$ ensures that no bacterial killing occurs, which is the reason for the strong negative correlation between $F$ and $B_{r r}$. Reverse mutation rates have little effect on the outcome. The positive correlation of $\alpha_{2}$ with respect to $B_{r r}$ is greater in magnitude at time $\mathrm{t}=360\left(\right.$ PRCC 0.59) than at time $\mathrm{t}=480$ (PRCC 0.12) because the concentration of $B_{r s}$ is higher at day 360 , when $A_{2}$ is first cycled into use. After 120 days, the $B_{r s}$ class has dwindled as a result of bacterial killing by $A_{2}$, so the impact of mutation rate $\alpha_{2}$ on the outcome value of $B_{r r}$ has decreased. Other parameters in Table 9 have a fairly predictable effect on the outcome value of $B_{r r}$ (growth rate, etc.). The PRCC's for $F$ and $r_{r r}$ with respect to $B_{r r}$ at time $\mathrm{t}=480$ indicate that the combination of a very low value for $F$ and very high value for $r_{r r}$ produces a large outcome value for $B_{r r}$, which was the case in each of the three model simulations discussed earlier in this chapter.

A particularly unexpected finding of the sensitivity analysis is that mutation, reverse mutation, and killing rates generally have less effect on the model outcome than patient inflow/outflow, growth rates, carrying capacity, and bacterial concentration in the typical incoming patient. The pdf's of some of the parameters identified by sensitivity analysis as very influential ones, such as $K$ and $\hat{B}_{\text {total }}$, are based on estimates of the possible range of bacterial concentration in patients. These estimates vary widely. The 
bacterial concentration in a single patient may range from $10^{4} \mathrm{CFU} / \mathrm{mL}$ to $10^{15} \mathrm{CFU} / \mathrm{mL}$ [68]. Edelstein-Keshet [5] notes that the difficulty in determining concentration per patient occurs with viral infections as well as bacterial, writing that "...total viral population...may range over several orders of magnitude in individual hosts." (p. 243) The variability is so large that these parameters tend to dominate the model outcome.

We now turn our attention to a discussion of the data set obtained from the clinical trial performed at Hennepin County Medical Center (HCMC). The task of matching the data to the model output has proven to be a difficult undertaking. In the clinical trial at HCMC, isolates were collected from all patients in the ICU approximately once a week. We are only concerned here with those isolates testing positive for $P$. aeruginosa. The isolates were tested for resistance or susceptibility to several antibiotics, including those being cycled (ciprofloxacin and piperacillin/tazobactam). The data set gives the whole number of isolates per data collection day and the results of tests for resistance on each isolate. (No information about daily antibiotic concentration is included in the data.) While the data measures number of isolates, the model measures bacterial concentration. Converting the data from the clinical trial from number of isolates to bacterial concentration involves a fair amount of speculation, for reasons discussed in the previous paragraph. In addition, data analysis has thus far been inconclusive in determining whether there are any cyclic patterns occurring as a result of antibiotic usage.

Nevertheless, for the purpose of comparison of the data and model output in this paper, we have attempted to convert the data from number of isolates per data collection day to bacterial concentration per data collection day. Each isolate was classified as a member of exactly one of the four bacterial subpopulations $B_{s s}, B_{r s}, B_{s r}$, or $B_{r r}$, depending on the results of the resistance testing. Then a plausible value was chosen for the bacterial concentration in each patient from which an isolate was taken. In this way bacterial concentrations for each of the four subpopulations were derived for the data collection days. No information is available for the days in which data collection did not occur. These bacterial concentrations were plotted with respect to time and are shown as discrete data points superimposed on a simulation of the model in Figure 5. For the model simulation in Figure 5, all parameter values were set at the means of their pdf's, 

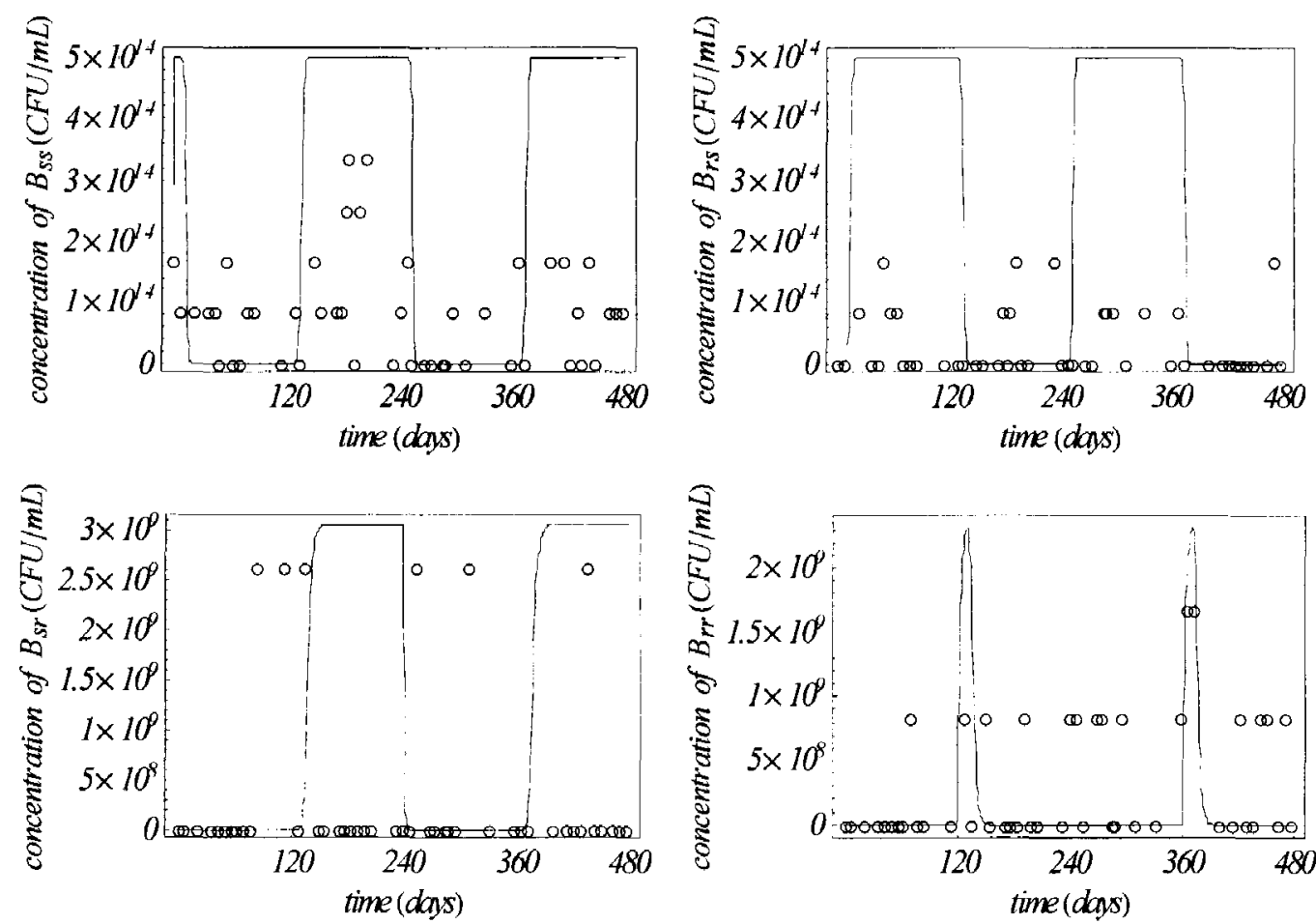

Figure 5 Comparison of Model Output and Data from Clinical Trial

and initial conditions were chosen according to data from day 1 of the clinical trial. Note that the change in initial conditions does not appear to change the behavior of the model. This is not surprising, as the initial condition for bacterial concentration was not identified as a significant input variable by the sensitivity analysis.

At this time the fit between the model and the data is not a good match. A second data set is currently being collected at Utrecht University Medical Center in Amsterdam. As we look ahead to continued work with the model, not only will more data become available, but the model parameter values (such as mutation and reverse mutation rates) will become available in the medical literature, as the problem of resistance is the focus of much research in the medical community today. The difficulty in estimating patient bacterial concentration also presents an obstacle that must be overcome before the model can be used in the clinical setting. Nevertheless, when both data sets are available for model comparison and parameter values can be assessed with less uncertainty, there will be a marked improvement in the fit between the model and the data. 
Further model revisions will also facilitate a better fit between the model and the data. The One Bug-Two Drug model currently classifies bacteria as either susceptible or resistant. However, it may be more realistic to incorporate some type of intermediate susceptible/resistant classification(s) into the model. In addition, the bacterial equations (3.7)-(3.10) do not consider specific antibiotic concentration as a contributing factor to resistance development beyond the simple on/off dynamics captured by the drug indicator function. The medical literature indicates that the gain of resistance is often concentration-dependent, Future model revisions may measure the complex interplay between antibiotic concentration and resistance development more precisely by including the fluctuating antibiotic concentration as a parameter in the bacterial equations.

The One Bug-Two Drug model is not ready for use as a predictive tool in the clinical setting at this point, but once the data has been matched to the model output, the model will be valuable in studying the effects of antibiotic cycling. For example, the model can be examined to determine the optimal cycle length to minimize resistance, or to examine the long-term effects of cycling. It can also be used to investigate other pathogenic bacteria, such as $S$. aureus, by redefining parameter values accordingly.

The study of the dynamics of bacterial gain and loss of resistance as a result of antibiotic use is an area of research with the potential to save lives. As our understanding of the principles of biomathematics grows, so will our ability to comprehend and accurately model the dynamics of resistance. Ian Stewart writes in [2] that "our current mathematical understanding of biology, admittedly, is fragmented, piecemeal, and open to dispute - just as it is for any new science... I believe there may be a new kind of mathematical theory out there in the intellectual darkness... if we can find it, it will greatly illuminate some of the big puzzles of biology - development, reproduction, ecosystem dynamics, evolution..." (pp. xiii, 245; emphasis in original). Perhaps the key to truly understanding the dynamics of resistance will be found after further work with the model presented in this paper, or perhaps it lies instead in mathematical tools that are only now in the earliest stages of development. In either case, the future is likely to hold promising developments in the ongoing battle against bacterial infection. 


\section{REFERENCES}

1. Quoted in Bell, E.T. Men of Mathematics p. 172. New York: Simon and Schuster, 1937.

2. Stewart, I. Life's Other Secret: the New Mathematics of the Living World. New York: Wiley \& Sons, 1998.

3. Devlin, K. The Math Gene: How Mathematical Thinking Evolved and Why Numbers are Like Gossip. Great Britain: Weidenfeld \& Nicolson, 2000.

4. Katz, V. Biology and mathematics. Focus: The Newsletter of the American Mathematical Association 2003 Feb, 23(2):10-13.

5. Edelstein-Keshet, L. Mathematical Models in Biology. Boston: McGraw-Hill, 1988.

6. Kingsland, S.E. Modeling Nature. Chicago: University of Chicago Press, 1985.

7. Lynch, S. Dynamical Systems with Applications using Maple. Boston: Birkhäuser, 2001.

8. May, R.M. Stability and Complexity in Model Ecosystems. New Jersey: Princeton University Press, 2001.

9. Blower, S.M. \& Dowlatabadi, H. Sensitivity and uncertainty analysis of complex models of disease transmission: an HIV model, as an example. International Statistical Review 1994, 62(2):229-243.

10. Levy, S.B. The Antibiotic Paradox: How Miracle Drugs are Destroying the Miracle. New York: Plenum Press, 1992.

11. Fick, R.B., ed. Pseudomonas aeruginosa the Opportunist: Pathogenesis and Disease. Boca Raton: CRC Press, 1993.

12. Gessard, C. (1984) Classics in Infectious Disease: On the Blue and Green Coloration that Appears on Bandages. Reviews of Infectious Diseases. 6:S775-S776.

13. Gessard, C. (1882) Sur les colorations bleue et verte des lignes à pansements. C.R. Acad. Sci. Serie D. 94:536-538. 
14. Boronin, A.M. Diversity of Pseudomonas plasmids: To what extent? FEMS Micobiol Lett 1992 Dec 15, 79(1-3): 461-467.

15. Campa, M., Bendinelli, M., \& Friedman, H., eds. Pseudomonas aeruginosa as an Opportunistic Pathogen. New York: Plenum Press, 1993.

16. Govan, J. TOBI: Reducing the impact of Pseudomonal infection. Hosp Med 2002 July, 63(7): 421-425.

17. Fridkin, S. \& Gaynes, R. Antimicrobial resistance in intensive care units. Clinics in Chest Medicine 1999, 20(2):303-316.

18. Landman, D., et al. Citywide clonal outbreak of multiresistant Acinetobacter baumannii and Pseudomonas aeruginosa: the preantibiotic era has returned. Arch Intern Med 2002 Jul 8; 162(13):1515-20.

19. Tenover, F. \& McGowan, J. Reasons for the emergence of antibiotic resistance. American Journal of the Medical Sciences 1996 Jan, 311(1): 9-16.

20. Gold, H. \& Moellering, R. Antimicrobial-drug resistance. New England Journal of Medicine 1996 Nov 7, 335(19):1445-53.

21. Giamerellou, H. \& Antoniadou, A. Antipseudomonal antibiotics. Medical Clinics of North America 2001 Jan, 85(1): 19-42.

22. Cunha, B. Pseudomonas aeruginosa: resistance and therapy. Seminars in Respiratory Infections 2002 Sept, 17(3): 231-239.

23. Salyers, A.A. \& Whitt, D.D. Bacterial Pathogenesis: a Molecular Approach. $2^{\text {nd }}$ ed. Washington, DC: ASM Press, 2002.

24. Sader, H.S., et al. Piperacillin/tazobactam: Evaluation of its in vitro activity against bacteria isolated in two Brazilian hospitals and an overview of its antibacterial activity, pharmacokinetic properties and therapeutic potential. Braz J Infect Dis 1998 Oct, 2(5):241-255.

25. Johnson, D., Biedenbach, D., \& Jones, R. Potency and antimicrobial spectrum update for piperacillin/tazobactam (2000): emphasis on its activity against resistant organism populations and generally untested species causing community-acquired respiratory tract infections. Diagnostic Microbiology and Infectious Disease 2002, 43:49-60. 
26. Sorgel, F. \& Kinzig, M. Pharmacokinetics and tissue penetration of piperacillin/tazobactam with particular reference to its potential in abdominal and soft tissue infections. Eur J Surg Suppl 1994, 573:39-44.

27. Hooper, D.C. Emerging mechanisms of fluoroquinolone resistance. Emerging Infectious Diseases 2001 March-April, 7(2):337-341.

28. Walker, R. The fluoroquinolones. Mayo Clin Proc 1999, 74:1030-1037.

29. Dudley, M. Pharmacodynamics and pharmacokinetics of antibiotics with special reference to the fluoroquinolones. Am J Med $1991 \mathrm{Dec} 30,91(6 \mathrm{~A}): 45 \mathrm{~S}-50 \mathrm{~S}$.

30. Bruinsma, N., et al. Antibiotic use and the prevalence of antibiotic resistance in bacteria from healthy volunteers in the Dutch community. Infection 2003, 31(1):914.

31. Bonhoeffer, S., Lipsitch, M., \& Levin, B.R. Evaluating treatment protocols to prevent antibiotic resistance. Proc Natl Acad Sci USA 1997 Oct, 94:12106-12111.

32. Levin, B.R. Minimizing potential resistance: a population dynamics view. Clinical Infectious Diseases 2001, 33(S3):S161-9.

33. Roberts, R.R., et al. The use of economic modeling to determine the hospital costs associated with nosocomial infections. Clinical Infectious Diseases 2003, 36:142432.

34. The Brooklyn Antibiotic Resistant Task Force. The cost of resistance: effect of resistance among Staphylococcus aureus, Klebsiella pneumoniae, Acinetobacter baumannil, and Pseudomonas aeruginosa on length of hospital stay. Infect Control Hosp Epidemiol 2002, 23:106-108.

35. Patzer, J., Dzierzanowska, D., \& Turner, P. Susceptibility patterns of Gram-negative bacteria from a Polish intensive care unit, 1997-2000. International Journal of Antimicrobial Agents 2002, 19:431-434.

36. CDC NNIS System. National nosocomial infections surveillance (NNIS) system report, data summary from January 1992 to June 2002, Issued August 2002. Am J Infect Control 2002, 30:458-75.

37. DiMartino, P., et al. Antibiotic resistance and virulence properties of Pseudomonas strains from mechanically vented patients with pneumonia in intensive care units: 
comparison with imipenem-resistant extra-respiratory tract isolates from uninfected patients. Microbes and Infection 2002, 4:613-620.

38. Lepper, P.M., et al. Consumption of imipenem correlates with $\beta$-lactam resistance in Pseudomonas aeruginosa. Antimicrobial Agents and Chemotherapy 2002 Sept, 46(9): 2920-2925.

39. Lesch, C.A., et al. Multi-hospital analysis of antimicrobial usage and resistance trends. Diagnostic Microbiology and Infectious Disease 2001, 41:149-154.

40. Monnet, D.L., et al. Antimicrobial use and resistance in eight U.S. hospitals: complexities of analysis and modeling. Infection Control and Hospital Epidemiology 1998 June, 19(6):388-394.

41. Paul, K., De, P.K., \& Bhattacharya, S. Comparative efficacy of fluoroquinolones, aminoglycosides, ureidopenicillins \& newer cephaolosporins against $P$ seudomonas species. Indian J Med Res [A] 1992 May, 95:136-138.

42. McGowan, J. Minimizing antimicrobial resistance in hospital bacteria: can switching or cycling drugs help? Infection Control 1986, 7(12):573-576.

43. Andersson, D.I. \& Levin, B.R. The biological cost of antibiotic resistance. Curr Opin Microbiol 1999, 2(5):489-493.

44. Raymond, D., et al. Impact of a rotating empiric antibiotic schedule on infectious mortality in an intensive care unit. Critical Care Medicine 2001 June, 29(6):11011108.

45. Smith, H. \& Waltman, P. The Theory of the Chemostat: Dynamics of Microbial Competition. New York: Cambridge University Press, 1995.

46. Codd, G.A., ed. Aspects of Microbial Metabolism and Ecology. New York: Academic Press, 1984.

47. Hsu, S.B., Li, Y., \& Waltman, P. Competition in the presence of a lethal external inhibitor. Mathematical Biosciences 2000, 167:177-199.

48. Patten, B.C., ed. Systems Analysis and Simulation in Ecology. New York: Academic Press, 1971.

49. Lenski, R. \& Hattingh, S. Coexistence of two competitors on one resource and inhibitor: a chemostat model based on bacteria and antibiotics. J. Theor. Biol. 1986, 122:83-93. 
50. Wigner, E.P. The unreasonable effectiveness of mathematics in the natural sciences. Communications in Pure and Applied Mathematics 1960, 13:1-14.

51. Hamby, D.H. A comparison of sensitivity analysis techniques. Health Physics 1995 Feb, 68(2):195-204.

52. McKay, M.D., Beckman, R.J., \& Conover, W.J. A comparison of three methods for selecting values of input variables in the analysis of output from a computer code. Technometrics 1979 May, 21(2):239-245.

53. Seaholm, S.K., Ackerman, E., \& Wu, S. Latin hypercube sampling and the sensitivity analysis of a Monte Carlo epidemic model. Int J Biomed Comput 1988, 23:97-112.

54. Iman, R., Helton, J., \& Campbell, J. An approach to sensitivity analysis of computer models: Part I - introduction, input variable selection and preliminary variable assessment. Journal of Quality Technology 1981 July, 13(3):174-183.

55. Swartzman, G.L. \& Kaluzny, S.P. Ecological Simulation Primer. New York: Macmillan, 1987.

56. O'Neill, R.V., Gardner, R.H., \& Mankin, J.B. Analysis of parameter error in a nonlinear model. Ecological Modelling 1980, 8:297-311.

57. Stitt, D.T., et al. Determination of growth rate of microorganisms in broth from oxygen-sensitive fluorescence plate reader measurements. Biotechniques 2002 , 32(3):684,686,688-9.

58. Gillespie, T. \& Masterton, R.G. Investigation into the selection frequency of resistant mutants and the bacterial kill rate by levofloxacin and ciprofloxacin in non-mucoid Pseudomonas aeruginosa isolates from cystic fibrosis patients. International Journal of Antimicrobial Agents 2002, 19:377-382.

59. Igumbor, E., et al. Antibiotic sensitivity and plasmid profiles of Pseudomonas aeruginosa. Cent Afr J Med 2000 Nov, 46(11): 296-300.

60. Burgess, D.S. \& Nathisuwan, S. Cefepime, piperacillin/tazobactam, gentamicin, ciprofloxacin, and levofloxacin alone and in combination against Pseudomonas aeruginosa. Diagnostic Microbiology and Infectious Disease 2002, 44:35-41.

61. Jhee, S.S., et al. Piperacillin-tazobactam pharmacokinetics in patients with intrabdominal infections. Pharmacotherapy 1995 Jul-Aug, 15(4):472-8. 
62. Garrelts, J.C., et al. Ciprofloxacin pharmacokinetics in burn patients. Antimicrob Agents Chemother 1996 May, 40(5):1153-6.

63. Hyatt, J.M., Nix, D.E., \& Schentag, J.J. Pharmacokinetics and pharmacodynamic activities of ciprofloxacin against strains of Streptococcus pneumoniae, Staphylococcus aureus, and Pseudomonas aeruginosa for which MIC's are similar. Antimicrob Agents Chemother 1994 Dec, 38(12): 2730-7.

64. Wolfram, S. The Mathematica Book. $4^{\text {th }}$ ed. Illinois: Wolfram Media, 1999.

65. Conover, W.J. Practical Nonparametric Statistics. New York: Wiley \& Sons, 1971.

66. Kendall, M. \& Stuart, A. The Advanced Theory of Statistics. Volume II. $4^{\text {th }}$ ed. New York: Macmillan, 1979.

67. Quoted in Lewin, R. Why is development so illogical? Science 1984 June, 224:1328.

68. Tsukayama, D.T. Personal correspondence. 2003. 


\title{
APPENDIX 1
}

\section{Latin Hypercube Sampling with Mathematica}

\author{
$\ll$ Statistics 'ContinuousDistributions' \\ $\ll$ DiscreteMath 'Combinatorica`
}

Unprotect [PDF, CDF, Quantile] ;

Clear[HDF, CDF, Quantile] ;

Off [NIntegrate: : ploss] ;

off [NIntegrate: : slwoon] ;

Off [NIntegrate: : ncub] ;

off [ FindRoot: :frmp] ;

FDF $[$ TriDist $[a, b], x]:=((4 *$ Unitstep $[x-a] *$ Unitstep $[b-x]) /(b-a) \wedge 2)$ * $((x-a) *$ UnitStep $[(a+b) / 2-x]+(b-x) *$ Unitstep $[x-(a+b) / 2])$

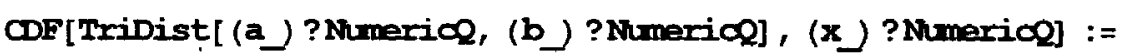
$B l o c k[\{t\}$, NIntegrate [RDF[TriDist $[a, b], t],\{t, a, x\}]]$

Quantile [dist: Tribist [ (a) ? Numerio, (b] ? Numeriog], (q) ? Numerico] := Which $[q \geq 1$. , $b, q \leq 0$. , a, True,

$\operatorname{Block}[\{x\}, x /$. FindRoot [CDF[dist, $x]-q,\{x,(2 a+b) / 3,(a+2 b) / 3\}$, Maxiterations $\rightarrow$ 100] ] ]

Protect [PDF, CDF, Quantile] ;

equiprob [dist_, $n]$ : = Partition [Table [Quantile[dist, $k / n],\{k, 0, n]], 2,1]$

distparam $[1]=\operatorname{TriDist}\left[6 * 10^{4}, 12 * 10^{7}\right]$;

distparam [2] $=$ TriDist $\left[10^{12}, 10^{15}\right]$;

distparam $[3]=$ TriDist $\left[10^{4}, 10^{7}\right]$;

distparam $[4]=$ TriDist $[36.97,45.36]$;

distparam $[5]=$ TriDist $[0.8 * 36.97,0.8 * 45.36]$;

distparam $[6]=$ TriDist $[0.8 * 36.97,0.8 * 45.36]$;

distparam $[7]=$ TriDist $[0.6 * 36.97,0.6 * 45.36]$;

distparam $[8]=$ TriDist $\left[5.82 * 10^{5}, 1.15 * 10^{6}\right]$;

distparam $[9]=$ TriDist $\left[3.125 * 10^{-7}, 6.25 * 10^{-6}\right]$;

distparam $[10]=$ TriDist $\left[1.5625 * 10^{-7}, 3.125 * 10^{-6}\right]$;

distparam $[11]=$ TriDist $\left[0.25 * 3.125 * 10^{-7}, 0.25 * 6.25 * 10^{-6}\right]$;

distparam $[12]=$ TriDist $\left[0.25 * 1.5625 * 10^{-7}, 0.25 * 3.125 * 10^{-6}\right]$; 


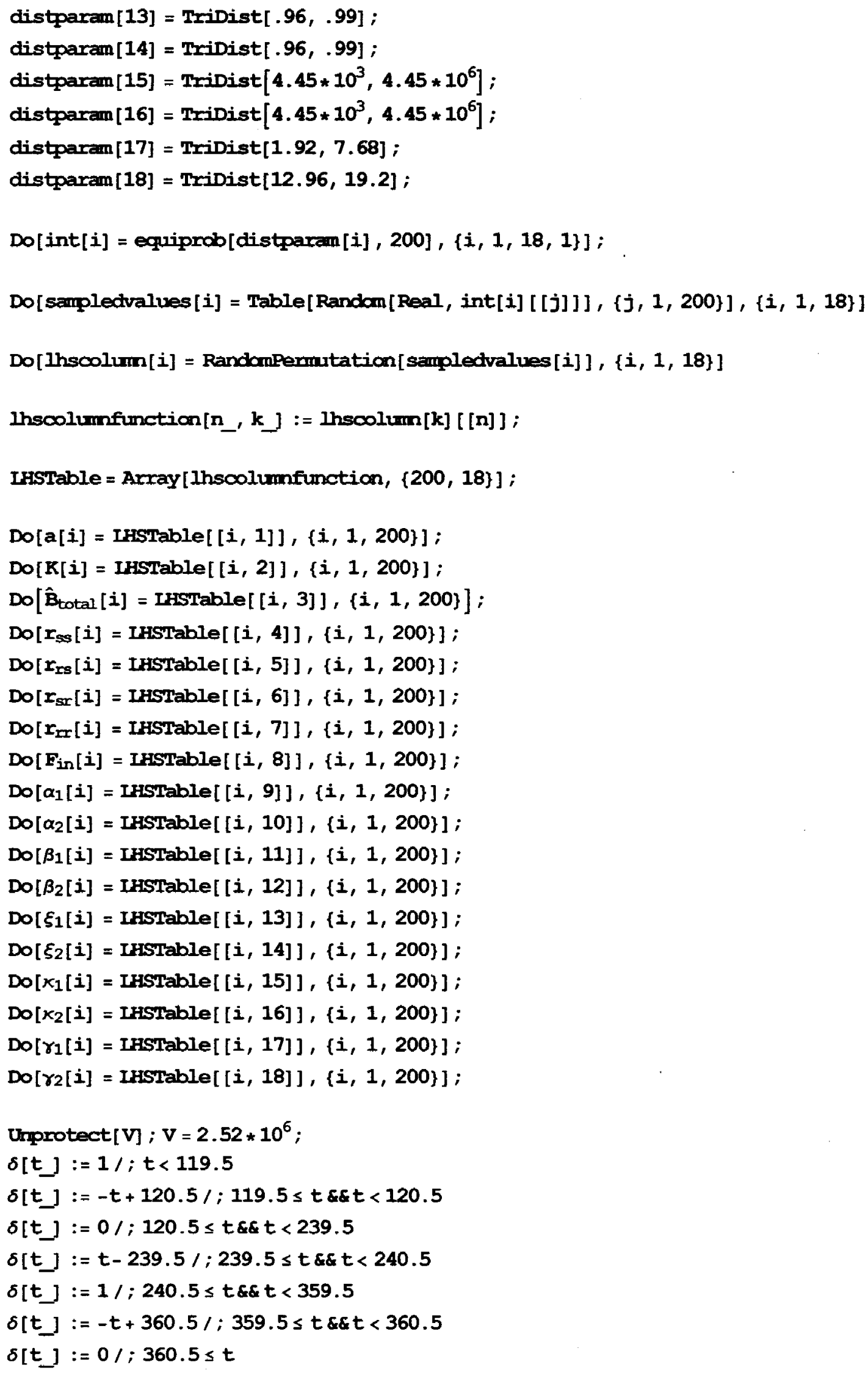


$\operatorname{ode} 1=y_{1}^{\prime}[t]=r_{s s}[i] * y_{1}[t] *\left(1.0-\left(y_{1}[t]+y_{2}[t]+y_{3}[t]+y_{4}[t]\right) /(K[i])\right)+$

$F_{i n}[i] * 0.8 * \hat{B}_{\text {total }}[i] / V-F_{\text {in }}[i] * Y_{1}[t] / V-\alpha_{1}[i] * \delta[t] * Y_{1}[t]-\alpha_{2}[i] *(1-\delta[t]) * Y_{1}[t]+$

$\beta_{1}[i] *(1-\delta[t]) * Y_{2}[t]+\beta_{2}[i] * \delta[t] * Y_{3}[t]-\xi_{1}[i] * \delta[t] * Y_{1}[t]-\xi_{2}[i] *(1-\delta[t]) * Y_{3}[t] ;$ ode2 $=Y_{2}{ }^{\prime}[t]=r_{r s}[i] * Y_{2}[t] *\left(1.0-\left(Y_{1}[t]+Y_{2}[t]+Y_{3}[t]+Y_{4}[t]\right) /(K[i])\right)+$

$F_{\text {in }}[i] * 0.09 * \hat{B}_{\text {totai }}[i] / V-F_{\text {in }}[i] * Y_{2}[t] / V+\alpha_{1}[i] * \delta[t] * Y_{1}[t]-\alpha_{2}[i] *(1-\delta[t]) * Y_{2}[t]-$

$\beta_{1}[i] *(1-\delta[t]) * Y_{2}[t]+\beta_{2}[i] * \delta[t] * Y_{4}[t]-\xi_{2}[i] *(1-\delta[t]) * Y_{2}[t] ;$

$\operatorname{ode} 3=Y_{3}{ }^{\prime}[t]==r_{\text {sr }}[i] * Y_{3}[t] *\left(1.0-\left(Y_{1}[t]+Y_{2}[t]+Y_{3}[t]+Y_{4}[t]\right) /(K[i])\right)+$

$F_{\text {in }}[i] * 0.09 * \hat{B}_{\text {total }}[i] / V-F_{i n}[i] * Y_{3}[t] / V-\alpha_{1}[i] * \delta[t] * Y_{3}[t]+\alpha_{2}[i] *(1-\delta[t]) * Y_{1}[t]+$

$\beta_{1}[i] *(1-\delta[t]) * Y_{4}[t]-\beta_{2}[i] * \delta[t] * Y_{3}[t]-\xi_{1}[i] * \delta[t] * Y_{3}[t] ;$

$\operatorname{ode} 4=Y_{4} \cdot[t]=x_{I x}[i] * Y_{4}[t] *\left(1.0-\left(Y_{1}[t]+Y_{2}[t]+Y_{3}[t]+Y_{4}[t]\right) /(R[i])\right)+$

$F_{i n}[i] * 0.02 * \hat{B}_{\text {total }}[i] / V-F_{\text {in }}[i] * Y_{4}[t] / V+\alpha_{1}[i] * \delta[t] \star Y_{3}[t]+\alpha_{2}[i] *(1-\delta[t]) \star Y_{2}[t]-$

$\beta_{1}[i] *(1-\delta[t]) * Y_{4}[t]-\beta_{2}[i] * \delta[t] * Y_{4}[t] ;$

ode5 $=Y_{5}{ }^{\prime}[t]==12 * 800 *\left(Y_{1}[t]+Y_{3}[t]\right) * \delta[t] /\left(x_{1}[i]+Y_{1}[t]+Y_{3}[t]\right)-\gamma_{1}[i] * Y_{5}[t] ;$

ode6 $=Y_{6}{ }^{\prime}[t]==12 * 13500 *\left(Y_{1}[t]+Y_{2}[t]\right) *(1-\delta[t]) /\left(\kappa_{2}[i]+Y_{1}[t]+Y_{2}[t]\right)-\gamma_{2}[i] * Y_{6}[t]$;

\section{solutiontable $=$}

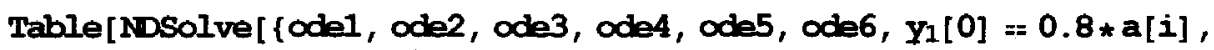

$\left.\mathrm{Y}_{2}[0]==0.09 * \mathrm{a}[\mathrm{i}], \mathrm{Y}_{3}[0]=0.09 * \mathrm{a}[\mathrm{i}], \mathrm{Y}_{4}[0]==0.02 \star \mathrm{a}[\mathrm{i}], \mathrm{Y}_{5}[0]==0, \mathrm{Y}_{6}[0]==0\right\}$,

$\left\{Y_{1}, Y_{2}, Y_{3}, Y_{4}, Y_{5}, Y_{6}\right\},\{t, 0,480\}$, MaxSteps $\left.\left.\rightarrow 10000000\right],\{i, 1,200,1\}\right]$;

finaloutcome $[1]=$ Table $\left[\mathrm{Y}_{1}[480] /\right.$. solutiontable $\left.\left.[i]\right],\{i, 1,200\}\right] / /$ Flatten

$5.50053 \times 10^{14}, 5.59588 \times 10^{14}, 7.15696 \times 10^{14}, 4.3064 \times 10^{14}, 4.17005 \times 10^{14}, 8.15753 \times 10^{14}$, $3.48684 \times 10^{14}, 4.13781 \times 10^{14}, 6.71623 \times 10^{14}, 7.16351 \times 10^{14}, 4.5463 \times 10^{14}, 5.74526 \times 10^{14}$, $3.66267 \times 10^{14}, 5.28508 \times 10^{14}, 6.78334 \times 10^{14}, 9.13357 \times 10^{14}, 4.27986 \times 10^{14}$,

$7.95678 \times 10^{14}, 4.25739 \times 10^{14}, 2.07992 \times 10^{14}, 7.1967 \times 10^{14}, 4.38733 \times 10^{14}, 7.35277 \times 10^{14}$, $1.9042 \times 10^{14}, 6.47758 \times 10^{14}, 3.95808 \times 10^{14}, 1.65245 \times 10^{14}, 6.09172 \times 10^{14}, 7.67298 \times 10^{14}$, $7.0808 \times 10^{14}, 6.38678 \times 10^{14}, 6.53665 \times 10^{14}, 6.12261 \times 10^{13}, 5.90041 \times 10^{14}, 8.25347 \times 10^{14}$, $3.72487 \times 10^{14}, 7.28863 \times 10^{14}, 6.30466 \times 10^{14}, 3.40144 \times 10^{14}, 8.00207 \times 10^{14}, 3.42234 \times 10^{14}$, $5.31612 \times 10^{14}, 6.70176 \times 10^{14}, 2.59663 \times 10^{14}, 6.27128 \times 10^{14}, 1.36092 \times 10^{14}, 5.55213 \times 10^{14}$, $5.83817 \times 10^{14}, 5.53829 \times 10^{14}, 3.06027 \times 10^{14}, 2.23083 \times 10^{14}, 1.43667 \times 10^{14}, 4.54995 \times 10^{14}$, $1.82373 \times 10^{14}, 2.22235 \times 10^{14}, 1.1515 \times 10^{14}, 2.91332 \times 10^{14}, 5.25 \times 10^{14}, 9.85409 \times 10^{14}$, $5.35757 \times 10^{14}, 6.18777 \times 10^{14}, 6.01427 \times 10^{14}, 5.09005 \times 10^{14}, 4.10351 \times 10^{14}, 5.15843 \times 10^{14}$, $5.89758 \times 10^{14}, 1.62071 \times 10^{14}, 4.58316 \times 10^{14}, 5.95425 \times 10^{14}, 2.31553 \times 10^{14}, 8.88801 \times 10^{14}$, $5.47324 \times 10^{14}, 4.20226 \times 10^{14}, 1.30787 \times 10^{14}, 7.28594 \times 10^{14}, 5.18844 \times 10^{14}, 8.38016 \times 10^{14}$, $6.33296 \times 10^{14}, 9.04889 \times 10^{14}, 7.84954 \times 10^{14}, 3.28639 \times 10^{14}, 4.84053 \times 10^{14}, 8.74729 \times 10^{14}$, $7.73112 \times 10^{14}, 3.50879 \times 10^{14}, 4.2257 \times 10^{14}, 8.32163 \times 10^{14}, 4.80703 \times 10^{14}, 4.89513 \times 10^{14}$, $6.44835 \times 10^{14}, 5.3406 \times 10^{14}, 3.22039 \times 10^{14}, 5.3888 \times 10^{14}, 4.42812 \times 10^{14}, 6.87621 \times 10^{14}$, $6.5212 \times 10^{14}, 4.75881 \times 10^{14}, 2.02964 \times 10^{14}, 8.12917 \times 10^{14}, 1.94734 \times 10^{14}, 7.04113 \times 10^{14}$, $5.98293 \times 10^{14}, 4.35344 \times 10^{14}, 1.73288 \times 10^{14}, 2.97472 \times 10^{14}, 4.99322 \times 10^{14}, 2.13828 \times 10^{14}$, $5.03811 \times 10^{14}, 3.88899 \times 10^{14}, 2.66682 \times 10^{14}, 4.94735 \times 10^{14}, 4.72336 \times 10^{14}, 3.75855 \times 10^{14}$, $7.90459 \times 10^{14}, 3.82761 \times 10^{14}, 3.80677 \times 10^{14}, 9.25709 \times 10^{14}, 5.03977 \times 10^{14}, 8.02264 \times 10^{13}$, $3.55455 \times 10^{14}, 2.39956 \times 10^{14}, 7.60826 \times 10^{14}, 6.62994 \times 10^{14}, 5.81652 \times 10^{14}$, $2.71427 \times 10^{14}, 3.69424 \times 10^{14}, 5.19328 \times 10^{14}, 6.05967 \times 10^{14}, 4.0798 \times 10^{14}, 7.56154 \times 10^{14}$, 
$5.42772 \times 10^{14}, 3.87513 \times 10^{14}, 4.03753 \times 10^{14}, 4.9324 \times 10^{14}, 4.76723 \times 10^{14}, 2.80497 \times 10^{14}$, $3.32405 \times 10^{14}, 6.89312 \times 10^{14}, 4.8814 \times 10^{14}, 4.45353 \times 10^{14}, 3.18004 \times 10^{14}, 2.76115 \times 10^{14}$, $6.4154 \times 10^{14}, 6.18401 \times 10^{14}, 4.65522 \times 10^{14}, 3.35362 \times 10^{14}, 3.59876 \times 10^{14}, 8.59901 \times 10^{14}$, $2.34852 \times 10^{14}, 3.19592 \times 10^{14}, 7.48889 \times 10^{14}, 5.62684 \times 10^{14}, 4.98853 \times 10^{14}, 5.97748 \times 10^{14}$, $8.67065 \times 10^{14}, 5.12822 \times 10^{14}, 5.67654 \times 10^{14}, 4.00575 \times 10^{14}, 5.76394 \times 10^{14}, 4.3616 \times 10^{14}$, $4.62553 \times 10^{14}, 6.96885 \times 10^{14}, 6.22999 \times 10^{14}, 5.22789 \times 10^{14}, 7.00567 \times 10^{14}, 3.08703 \times 10^{14}$, $2.83308 \times 10^{14}, 2.86543 \times 10^{14}, 6.83754 \times 10^{14}, 1.04249 \times 10^{14}, 5.09799 \times 10^{14}, 7.81381 \times 10^{14}$, $2.44667 \times 10^{14}, 7.38504 \times 10^{14}, 3.91334 \times 10^{14}, 4.14099 \times 10^{13}, 1.54353 \times 10^{14}, 2.99933 \times 10^{14}$, $6.59013 \times 10^{14}, 7.49296 \times 10^{14}, 8.46975 \times 10^{14}, 5.74529 \times 10^{14}, 4.49627 \times 10^{14}$, $3.99963 \times 10^{14}, 2.49516 \times 10^{14}, 4.69938 \times 10^{14}, 3.6391 \times 10^{14}, 6.11938 \times 10^{14}, 5.63661 \times 10^{14}$, $5.69265 \times 10^{14}, 5.43594 \times 10^{14}, 4.81762 \times 10^{14}, 3.45765 \times 10^{14}, 6.77045 \times 10^{14}$, $4.62251 \times 10^{14}, 2.57342 \times 10^{14}, 9.86302 \times 10^{13}, 3.12828 \times 10^{14}, 4.49498 \times 10^{14}, 6.6451 \times 10^{14}$,

\section{DispersionReport [finaloutoome [1] ]}

$\left\{\right.$ Variance $\rightarrow 4.121 \times 10^{28}$, StandardDeviation $\rightarrow 2.03002 \times 10^{14}$, SampleRange $\rightarrow 9.43999 \times 10^{14}$, MeanDeviation $\rightarrow 1.65303 \times 10^{14}$, MedianDeviation $\rightarrow 1.46978 \times 10^{14}$, QuartileDeviation $\rightarrow 1.46703 \times 10^{14}$ \}

\section{Min [finaloutcome[1] ]}

$4.14099 \times 10^{13}$

\section{Max [finaloutcame [1] ]}

$9.85409 \times 10^{14}$

\section{Man[finaloutoome[1]]}

$4.96501 \times 10^{14}$

\section{finaloutoome[2] = Table[Y2[480] / solutiontable[ [i] ], $\{i, 1,200]] / /$ Flatten}

\{134729., 213515., 230276., 178944., 148268., 33641.2, 256706., 199911., 85994.2, 154129., $194791 ., 116074 ., 197299 ., 60846.3,82592.7,91378.3,82469.2,240612 ., 214952 .$, $154710 ., 190974 ., 66020.8,154443 ., 93244.8,76239.3,253652 ., 96966.1,73166.7$, $149118 ., 64850.9,25313.3,128511 ., 184212 ., 155824 ., 153902 ., 141186 ., 136316 .$, $92090.8,148619 ., 114605 ., 123418 ., 170531 ., 85751.3,250260 ., 83441 ., 144470 ., 190399 .$, 250775., 162909., 215940., 210426., 223272., 130639., 120955., 126445., 177456., $118469 ., 145732 ., 48254$, 199285., 118426., 161740., 246782., 201983., 48409.9, 142720., 148812., 238305., 154806., 23483.1, 164346., 143114., 130383., 168739., $150202 ., 276477 ., 93618.5,174888 ., 104087 ., 178127 ., 163852$., 143007., 113925., $75592.2,61261.5,153824 ., 63759.6,46469.8,110791 ., 166864 ., 97264.6,149994 .$, $178251 ., 83590.5,140239 ., 108073 ., 243333 ., 130331 ., 125584 ., 196247 ., 126286 .$, 155174., 12603.1, 181306., 270885., 138341., 47276.1, 116795., 178998., 278190., 247596., 150674., 266804., 125022., 162225., 214366., 103849., 69294.2, 121553., 180189., 164333., 217585., 99932.7, 171426., 66973.4, 50044.1, 188683., 78884.9, $126135 ., 225877 ., 195839 ., 82218.2,95034.6,166948 ., 152229 ., 185924 ., 139631 .$, 254965., 88860.1, 100259., 148526., 98174.3, 29878.4,81536.1, 48477.1, 214047., $159161 ., 161092 ., 145900 ., 275688 ., 64213.3,219462 ., 170163 ., 111826 ., 83641.5$, 
$103402 ., 271911 ., 146984 ., 67347.9,201383 ., 39371.9,190664 \ldots, 130736 ., 235405 .$, $134648 ., 214568 ., 115859 ., 217334$, 124534., 231625., 107730., 147608., 164201., $134206 ., 159219 ., 118053 ., 117931 ., 280810$., 151054., 205758., 215613., 13338.9, $115291 ., 233286 ., 223429 ., 138397 ., 65240.7,168507 ., 200794 ., 144650 ., 87288 .$, 152459., 152390., 129721., 188001., 148479., 291951., 74342.1, 184295., 111262.\}

\section{DispersionReport [fingloutocme [2] ]}

(Variance $\rightarrow 3.74766 \times 10^{9}$, StandardDeviation $\rightarrow 61218.2$, SampleRange $\rightarrow 279348$., MeanDeviation $\rightarrow 48687.6$, MedianDeviation $\rightarrow$ 42593.3, QuartileDeviation $\rightarrow 42358.3$ \}

\section{Min[finaloutoome[2] ]}

12603.1

\section{Max [finaloutocme[2] ]}

291951.

\section{Mean[finaloutoome[2] ]}

147168 .

\section{finaloutcome[3] = Table $\left[Y_{3}[480] /\right.$. solutiontable $\left.\left.[i]\right],\{i, 1,200\}\right] / /$ Flatten}

$\left\{2.26559 \times 10^{10}, 1.64634 \times 10^{10}, 2.0052 \times 10^{10}, 1.4082 \times 10^{10}, 1.0457 \times 10^{10}, 1.17063 \times 10^{10}\right.$, $7.664 \times 10^{9}, 1.50105 \times 10^{10}, 1.54466 \times 10^{10}, 1.43269 \times 10^{10}, 1.28353 \times 10^{10}, 1.88607 \times 10^{10}$, $9.45245 \times 10^{9}, 1.29438 \times 10^{10}, 1.9514 \times 10^{10}, 2.63703 \times 10^{10}, 1.0305 \times 10^{10}, 2.96109 \times 10^{10}$, $7.67436 \times 10^{9}, 4.70097 \times 10^{9}, 1.01319 \times 10^{10}, 1.4878 \times 10^{10}, 1.57539 \times 10^{10}, 3.80977 \times 10^{9}$, $2.40861 \times 10^{10}, 1.28644 \times 10^{10}, 4.01399 \times 10^{9}, 6.54963 \times 10^{9}, 1.7186 \times 10^{10}, 2.35406 \times 10^{10}$, $2.50834 \times 10^{10}, 9.17995 \times 10^{9}, 2.0478 \times 10^{9}, 1.76665 \times 10^{10}, 5.55608 \times 10^{10}, 6.38529 \times 10^{10}$, $2.0009 \times 10^{10}, 4.55657 \times 10^{9}, 5.77666 \times 10^{9}, 1.67649 \times 10^{10}, 3.03853 \times 10^{9}, 9.89338 \times 10^{9}$, $1.64197 \times 10^{10}, 7.46891 \times 10^{9}, 2.76626 \times 10^{10}, 7.24047 \times 10^{9}, 1.2282 \times 10^{10}, 1.7975 \times 10^{10}$, $1.84025 \times 10^{10}, 2.8432 \times 10^{9}, 3.85072 \times 10^{9}, 4.91776 \times 10^{9}, 6.25738 \times 10^{9}, 5.33367 \times 10^{9}$, $3.39728 \times 10^{9}, 1.18013 \times 10^{9}, 6.35388 \times 10^{9}, 1.45926 \times 10^{10}, 1.94426 \times 10^{10}, 9.3172 \times 10^{9}$, $1.66926 \times 10^{10}, 9.52058 \times 10^{9}, 2.682 \times 10^{10}, 2.06138 \times 10^{10}, 1.24926 \times 10^{10}, 1.5922 \times 10^{10}$, $1.71736 \times 10^{9}, 1.81394 \times 10^{10}, 1.81934 \times 10^{10}, 5.9144 \times 10^{9}, 1.51002 \times 10^{10}, 5.14161 \times 10^{9}$, $4.52726 \times 10^{9}, 2.19076 \times 10^{9}, 9.20365 \times 10^{9}, 2.50559 \times 10^{9}, 1.09147 \times 10^{10}, 1.22399 \times 10^{10}$, $3.40131 \times 10^{10}, 4.52826 \times 10^{9}, 7.36786 \times 10^{9}, 2.96841 \times 10^{10}, 2.70207 \times 10^{10}, 2.33597 \times 10^{10}$, $9.29984 \times 10^{9}, 1.50272 \times 10^{10}, 6.81547 \times 10^{9}, 4.85879 \times 10^{9}, 1.60902 \times 10^{10}, 1.14432 \times 10^{10}$, $1.12631 \times 10^{10}, 6.58322 \times 10^{9}, 1.14978 \times 10^{10}, 5.42128 \times 10^{9}, 1.97959 \times 10^{10}, 1.83255 \times 10^{10}$, $7.45745 \times 10^{9}, 4.81822 \times 10^{9}, 3.17555 \times 10^{10}, 3.76574 \times 10^{9}, 1.97066 \times 10^{10}, 6.54745 \times 10^{9}$, $1.2858 \times 10^{10}, 6.24058 \times 10^{9}, 6.16682 \times 10^{9}, 1.36374 \times 10^{10}, 2.90928 \times 10^{9}, 8.66542 \times 10^{9}$, $8.39336 \times 10^{9}, 5.20671 \times 10^{9}, 5.24967 \times 10^{9}, 1.06429 \times 10^{10}, 8.55015 \times 10^{9}, 1.11324 \times 10^{10}$, $1.17406 \times 10^{10}, 1.02585 \times 10^{10}, 2.02928 \times 10^{10}, 1.11663 \times 10^{10}, 1.59821 \times 10^{9}$,

$7.30849 \times 10^{9}, 6.04876 \times 10^{9}, 5.03035 \times 10^{10}, 5.35173 \times 10^{9}, 2.46833 \times 10^{10}, 5.73448 \times 10^{9}$, $5.46227 \times 10^{9}, 1.22007 \times 10^{10}, 1.30057 \times 10^{10}, 7.20972 \times 10^{9}, 1.87193 \times 10^{10}, 7.01399 \times 10^{9}$, $1.43338 \times 10^{10}, 5.00436 \times 10^{9}, 2.62901 \times 10^{10}, 2.84429 \times 10^{10}, 8.552 \times 10^{9}, 7.16429 \times 10^{9}$, $2.87873 \times 10^{10}, 1.67299 \times 10^{10}, 9.66717 \times 10^{9}, 9.93059 \times 10^{9}, 7.06989 \times 10^{9}, 2.40542 \times 10^{10}$, $1.68072 \times 10^{10}, 4.25695 \times 10^{9}, 1.64736 \times 10^{10}, 7.57549 \times 10^{9}, 1.79308 \times 10^{10}, 2.42491 \times 10^{10}$, $1.88891 \times 10^{10}, 2.69111 \times 10^{10}, 1.61623 \times 10^{10}, 1.31225 \times 10^{10}, 4.51919 \times 10^{10}$, 
$1.67984 \times 10^{10}, 7.63062 \times 10^{9}, 1.26611 \times 10^{10}, 8.55538 \times 10^{9}, 1.95216 \times 10^{10}, 8.59597 \times 10^{9}$, $2.59851 \times 10^{10}, 2.41891 \times 10^{10}, 9.79205 \times 10^{9}, 1.70948 \times 10^{10}, 1.21317 \times 10^{10}$, $8.63055 \times 10^{9}, 1.92768 \times 10^{9}, 5.84007 \times 10^{9}, 5.35043 \times 10^{9}, 1.82177 \times 10^{9}, 1.23517 \times 10^{10}$, $3.13505 \times 10^{10}, 4.17692 \times 10^{9}, 1.00121 \times 10^{10}, 6.10346 \times 10^{9}, 2.74216 \times 10^{9}, 6.71412 \times 10^{9}$, $1.19106 \times 10^{10}, 1.41089 \times 10^{10}, 2.42659 \times 10^{10}, 2.31624 \times 10^{10}, 1.65293 \times 10^{10}$, $1.28581 \times 10^{10}, 4.30904 \times 10^{10}, 1.68643 \times 10^{10}, 1.91113 \times 10^{10}, 9.29937 \times 10^{9}, 9.99234 \times 10^{9}$, $1.58248 \times 10^{10}, 7.90921 \times 10^{9}, 1.95818 \times 10^{9}, 5.83029 \times 10^{9}, 6.05545 \times 10^{9}, 1.2075 \times 10^{10}$, $\left.2.1645 \times 10^{10}, 4.50611 \times 10^{9}, 1.96731 \times 10^{9}, 5.28604 \times 10^{9}, 1.25041 \times 10^{10}, 7.02217 \times 10^{9}\right)$

\section{DispersionReport [ finaloutoome[3] ]}

\{Variance $\rightarrow 9.38132 \times 10^{19}$, StandardDeviation $\rightarrow 9.68572 \times 10^{9}$, SampleRange $\rightarrow 6.29185 \times 10^{10}$, MeanDeviation $\rightarrow 7.1197 \times 10^{9}$, MedianDeviation $\rightarrow 5.42527 \times 10^{9}$, QuartileDeviation $\rightarrow 5.74653 \times 10^{9}$ \}

\section{Min [finaloutcome [3] ]}

$1.18013 \times 10^{9}$

\section{Max [finaloutocme[3]]}

$6.40986 \times 10^{10}$

\section{Mean[finaloutoome [3] ]}

$1.33624 \times 10^{10}$

\section{finaloutcome [4] $=$ Table $\left[Y_{4}[480] /\right.$. solutiontable $\left.[[i]],\{i, 1,200\}\right] / /$ Flatten}

$\left\{2.91357 \times 10^{6}, 1.81494 \times 10^{7}, 954501 ., 304486 ., 2.77112 \times 10^{6}, 230913 ., 376932 ., 4.63282 \times 10^{6}\right.$, $111234 ., 478562 ., 393360 ., 292705 ., 3.08387 \times 10^{6}, 356872 ., 203280 ., 2.42908 \times 10^{7}$, $124222 ., 1.17581 \times 10^{7}, 331125 ., 486830 ., 308884 ., 2.34734 \times 10^{6}, 283950 ., 160433 \ldots$ $867326.1 .17491 \times 10^{6}, 145003 ., 455334.1 .80598 \times 10^{6}, 1.30146 \times 10^{6}, 8.00414 \times 10^{6}$, $311501 ., 1.62271 \times 10^{6}, 658783 ., 1.38251 \times 10^{7}, 1.92631 \times 10^{11}, 1.15565 \times 10^{6}, 157370$. , $1.08693 \times 10^{7}, 5.59774 \times 10^{6}, 1.59846 \times 10^{6}, 790526 ., 1.59926 \times 10^{6}, 766050 ., 3.15921 \times 10^{7}$, $1.74592 \times 10^{6}, 419684 ., 3.02161 \times 10^{6}, 890424 ., 364931 ., 1.39639 \times 10^{8}, 372039 ., 665683 .$, $8.17632 \times 10^{6}, 3.38029 \times 10^{6}, 310353 ., 2.69313 \times 10^{6}, 1.97623 \times 10^{6}, 578365 ., 268689 .$, $1.47967 \times 10^{7}, 237600 ., 796051 ., 1.57859 \times 10^{7}, 3.27551 \times 10^{6}, 1.04386 \times 10^{7}, 223532 .$, $1.50187 \times 10^{6}, 1.14146 \times 10^{6}, 285351 ., 479508 ., 322571 ., 223866 ., 326503 ., 952523 .$, $549197 ., 212667 ., 3.84563 \times 10^{6}, 5.45904 \times 10^{8}, 669315 ., 498659 ., 1.85392 \times 10^{6}$, $9.51603 \times 10^{8}, 2.79154 \times 10^{6}, 484238 ., 1.9396 \times 10^{7}, 85095.8,61245.4,338153 .$, $324326 ., 1.68614 \times 10^{6}, 214495 ., 740411 ., 4.0551 \times 10^{6}, 1.20057 \times 10^{6}, 1.28906 \times 10^{6}$, $384527 ., 180713 ., 1.89466 \times 10^{6}, 335721 ., 225113 ., 651032 ., 8.48039 \times 10^{6}, 487877$. , $1.80457 \times 10^{6}, 3.03925 \times 10^{6}, 122680,1.25401 \times 10^{6}, 276625 ., 360845,, 474643$. , $304128 ., 515820 ., 184264 ., 1.92708 \times 10^{7}, 280922 ., 176731 ., 133591 ., 179780 .$, $534923 ., 9.96093 \times 10^{9}, 6.37336 \times 10^{10}, 1.81206 \times 10^{10}, 4.0873 \times 10^{6}, 151849 ., 93385.1$, $1.06453 \times 10^{6}, 311267 ., 8.16715 \times 10^{9}, 389690,1.01564 \times 10^{6}, 5.77232 \times 10^{10}, 130303 .$, $7.07124 \times 10^{6}, 6.97619 \times 10^{7}, 342879 ., 924301 ., 501575 ., 261136 ., 163145 ., 3.09752 \times 10^{6}$, $2.07063 \times 10^{6}, 2.86134 \times 10^{9}, 792288 ., 93679.9,4.78279 \times 10^{6}, 259469 ., 8.99844 \times 10^{6}$, $5.94641 \times 10^{8}, 1.26439 \times 10^{7}, 315679 ., 4.84296 \times 10^{6}, 286665 ., 2.60537 \times 10^{7}, 172923 .$, $166955 ., 710447 ., 3.47536 \times 10^{6}, 285897 ., 1.05568 \times 10^{6}, 4.32758 \times 10^{8}, 3.01484 \times 10^{6}$, 
$182150 ., 1.47717 \times 10^{6}, 191446 ., 2.83417 \times 10^{7}, 269928 ., 314331 ., 235861 ., 532555 .$, $7.53978 \times 10^{7}, 1.28103 \times 10^{7}, 491949 ., 181585 ., 190793 ., 331555 ., 1.48737 \times 10^{6}, 676512$. , $714411,7.24329 \times 10^{6}, 578631,7.88469 \times 10^{6}, 371738,6.69365 \times 10^{7}, 3.56316 \times 10^{6}$, $2.29532 \times 10^{7}, 5.14928 \times 10^{6}, 535175 ., 316840 ., 1.19636 \times 10^{8}, 578914 ., 373618 .$, $\left.782934 ., 704079 ., 376082 ., 1.2221 \times 10^{6}, 394411 ., 5.46545 \times 10^{6}, 4.80723 \times 10^{6}, 243736.\right\}$

\section{DispersionReport [finaloutoome [4] ]}

\{Variance $\rightarrow 3.18424 \times 10^{20}$, StandardDeviation $\rightarrow 1.78444 \times 10^{10}$, SampleRange $\rightarrow 2.03706 \times 10^{11}$, MeanDeviation $\rightarrow 4.50843 \times 10^{9}$, MedianDeviation $\rightarrow 530562$., QuartileDeviation $\rightarrow 1.69679 \times 10^{6}$ \}

\section{Min [finaloutocme[4] ]}

61245.4

\section{Max [finaloutoome[4] ]}

$2.03707 \times 10^{11}$

\section{Mean [finaloutocme [4] ]}

$2.35697 \times 10^{9}$

\section{finaloutoome[5] = Table[Y5[480] / . solutiontable[ [i] ], \{i, 1, 200\}] // Flatten; Chop [finaloutoome [5] ]}

$\{0,0,0,0,0,0,0,0,0,0,0,0,0,0,0,0,0,0,0,0,0,0,0,0,0,0,0,0$, $0,0,0,0,0,0,0,0,0,0,0,0,0,0,0,0,0,0,0,0,0,0,0,0,0,0,0,0$, $0,0,0,0,0,0,0,0,0,0,0,0,0,0,0,0,0,0,0,0,0,0,0,0,0,0,0,0$, $0,0,0,0,0,0,0,0,0,0,0,0,0,0,0,0,0,0,0,0,0,0,0,0,0,0,0,0,0$, $0,0,0,0,0,0,0,0,0,0,0,0,0,0,0,0,0,0,0,0,0,0,0,0,0,0,0,0,0$, $0,0,0,0,0,0,0,0,0,0,0,0,0,0,0,0,0,0,0,0,0,0,0,0,0,0,0,0,0$, $0,0,0,0,0,0,0,0,0,0,0,0,0,0,0,0,0,0,0,0,0,0,0,0,0,0,0,0,0\}$

\section{finaloutcome [6] = Table [Y6[480] / solutiontable[ [i]] , [i, 1, 200\}] // Flatten}

(9695.89, 9853.78, 8894.89, 10808.6, 10124.5, 9028.92, 12195.5, 10097.7, 9639.27, 10495.8, $10270.1,11518.4,10439.3,9371.11,9017.06,10607.6,11687 ., 10634.9,10045.5$, $9904.26,9178.45,11114.6,8635.53,9866.43,9719.25,9983.07,9099.43,10326.9$, $10149.1,9659.27,10858.8,9799.85,10177.1,10013 ., 10381.9,9451.38,8787.64$, $8740.07,11587.6,11088.7,9743.85,10028.9,9843.1,10089.2,9556.16,11162.4$, $9721.93,11302.5,10254.7,10565.7,8983.52,9758.86,11402.8,9229.05,10245.4$, $10523.2,11802.2,10110.5,11322.3,11723.9,10671 ., 12298.4,9665.69,10293.8$, $8677.92,9134.79,8558.56,10446.7,10976.5,9877.58,9483 ., 10165 ., 9572.18,9414.55$, $10823.4,9427.14,9383.53,9544 ., 9465.15,9150.91,10364.6,10685.6,10281.6$, $9293.18,10301.9,9949.64,10039 ., 10699.1,11150.2,9589.21,11881.1,9261.86$, $10204.8,9088.58,10795 ., 11085.7,10122 ., 10761.5,8868.46,10471.3,11272.6$, $9680.48,11472.5,10428.3,8784.21,9787.03,10959.8,10317.9,9516.82,11826.3$, $9520.77,12004.7,9707.9,10918.7,9198.35,9622.57,10189.9,9970.92,9308.39$, $8931.56,11639.6,9834.1,10004.8,9899.24,9993.35,9064.8,9477.43,9121.79$, $11212.5,9597.25,9776.68,9566.13,9442.89,10867.7,10219.6,10377.2,8890.61$, 10934 , 10737.4, 10404.5, 11462.1,9957.23, 9941.42, 11190.5, 10659.1, 10135.5, 
$10075.8,11341.8,10060 ., 9823.71,8941.9,10023.9,10590.7,9606.68,9054.33$, $9287.97,10065.1,11248.1,10349.8,11949.3,8839.58,9505.93,9335.7,10339.4$, $10235.7,9649.39,9237.3,11399.9,9201.34,10402.4,10515.9,8996.88,11050.8$, $9329.64,10551.5,9916.75,11027.3,9734.87,11538.3,10467.9,10986.9,8720.21$, $9260.88,11014.7,10722.5,9816.42,10846.1,10638.9,9774.69,10605.1,10895.9$, $10174.4,10490.9,9890.23,9931.69,10767.6,10539.6,10209.9,9351.82,9401.13$ \}

\section{DispersionReport [finaloutoome[6]]}

$\{$ Variance $\rightarrow 664000$. , StandardDeviation $\rightarrow$ 814.862, SampleRange $\rightarrow 3739.84$, MeanDeviation $\rightarrow 660.713$, MedianDeviation $\rightarrow 566.473$, QuartileDeviation $\rightarrow 572.968\}$

Min [finaloutoome[6] ]

8558.56

\section{Max [finaloutocme [6] ]}

12298.4

\section{Maan [finaloutocme [6] ]}

10139.

Do[rankcolumn[j] = Table[Position[sarmpledvalues [j] , thscolumn[j] [i] ] ] , $\{i, 1,200\}] / /$ Flatten, $\{j, 1,18\}]$

rankoolumnfunction $[\mathrm{n}, \mathrm{k}]$ : = rankoolumn[k] [ [n] ]

rankmatrix = Array [rankoolumnfunction, $\{200,18\}]$;

orderedfinaloutocme [1] = Sort [finaloutcome [1] ] ;

rankcolumn [19] =

Table [Position [orderedfinaloutoome[1] , finaloutoome[1] [ [i] ] ] , \{i, 1, 200\}] // Flatten; rankmatxixiB $B_{s s}=$ Array $[$ rankoolumfunction, $\{200,19\}]$;

$\mathbf{T R}_{\mathrm{ss}}=$ Transpose [rankmatrixB $\mathbf{B}_{\mathrm{ss}}$ ] ;

$$
\begin{aligned}
& D O\left[c[i, j]=\left(\sum_{t=1}^{200}\left(\mathrm{~TB}_{s s}[[i, t]]-201 / 2\right) *\left(\mathrm{HB}_{s s}[[j, t]]-201 / 2\right)\right) /\right. \\
& \sqrt{\left(\sum_{t=1}^{200}\left(\mathrm{~TB}_{s s}[[i, t]]-201 / 2\right)^{2}\right) *\left(\sum_{s=1}^{200}\left(\mathrm{HB}_{s s}[[j, s]]-201 / 2\right)^{2}\right)},\{i, 1,18\},
\end{aligned}
$$

$\{j, 1,18\}]$

$\operatorname{Do}\left[c[i, 19]=\left(\sum_{t=1}^{200}\left(\operatorname{TB}_{s s}[[i, t]]-201 / 2\right) *\left(\operatorname{TB}_{s s}[[19, t]]-201 / 2\right)\right) /\right.$

$\left.\sqrt{ }\left(\left(\sum_{t=1}^{200}\left(\operatorname{TB}_{s s}[[i, t]]-201 / 2\right)^{2}\right) *\left(\sum_{s=1}^{200}\left(\operatorname{TB}_{s s}[[19, s]]-201 / 2\right)^{2}\right)\right),\{i, 1,18\}\right]$

$D o[c[19, i]=c[i, 19],\{i, 1,18\}]$

$c[19,19]=1$;

Umprotect $[C] ; C 1=\operatorname{Array}[C,\{19,19\}]$; 
$\mathrm{B} 1=$ Inverse $[\mathrm{Cl} / / \mathrm{N}]$;

$\mathrm{DO}\left[\gamma_{\mathrm{ss}}[i]=-\mathrm{B} 1[[i, 19]] / \sqrt{\mathrm{B} 1[[i, i]] * \mathrm{~B} 1[19,19]]} / / \mathrm{N},\{i, 1,14\}\right]$

PRCCinputparamsandiBs $=$ Table $\left[\gamma B_{s s}[i],\{i, 1,14\}\right]$

$\{-0.0776149,0.999989,-0.0656751,0.0984684,0.00416987,-0.0728436,0.0555663$,

$-0.319124,0.0304413,0.0736761,-0.0819341,0.103606,-0.0282147,-0.0160582\}$

$D o\left[t B_{s s}[i]=\gamma B_{s s}[i] * \sqrt{\frac{198}{1-\gamma B_{s s}[i] * \gamma B_{s s}[i]}} / / N,\{i, 1,14\}\right]$

tstatisticsB $B_{s s}=$ Table $\left[\right.$ tB $\left._{s s}[i],\{i, 1,14\}\right]$

$\{-1.09544,2939.79,-0.92613,1.39234,0.0586758,-1.02773,0.783097$,

$-4.73822,0.428546,1.03954,-1.15681,1.46576,-0.397173,-0.225988\}$

$\operatorname{Do}\left[\right.$ siglevell [i] $=2-2 * \operatorname{CDF}\left[\operatorname{StudentIDistribution}[198], \operatorname{Sign}\left[t \mathrm{~B}_{s s}[i]\right] * t B_{s s}[i]\right]$,

$\{i, 1,14\}]$

significancelevelsFrocSandB $\mathrm{ss}_{\mathrm{ss}}=$ Table [siglevell $\left.[i],\{i, 1,14\}\right]$

$\left\{0.274654,2.485700857820 \times 10^{-461}, 0.355506,0.165381,0.95327,0.305331,0.434506\right.$,

$\left.4.10863 \times 10^{-6}, 0.668719,0.299821,0.248746,0.144301,0.691667,0.821444\right\}$

orderedfinaloutocme [2] = Sort [finaloutoome [2] ] ;

rankcolumn[19] $=$

Table[Position[orderedfinaloutocme[2], finaloutcome[2] [[i] ] ], \{i, 1, 200\}] // Flatten;

rankmatrixB $B_{r s}=$ Array $[$ rankcolumnfunction, $\{200,19\}]$;

$\mathbf{H B}_{\mathrm{IS}}=$ Transpose $\left[\right.$ rankmatrix $\mathbf{B}_{\mathrm{IS}}$ ] ;

$\operatorname{Do}\left[C 2[i, j]=\left(\sum_{t=1}^{200}\left(\operatorname{TB}_{r s}[[i, t]]-201 / 2\right) *\left(\operatorname{LB}_{r s}[[j, t]]-201 / 2\right)\right) /\right.$

$\sqrt{\left(\sum_{t=1}^{200}\left(\mathrm{HB}_{\mathrm{rs}}[[i, t]]-201 / 2\right)^{2}\right) *\left(\sum_{s=1}^{200}\left(\mathrm{HB}_{\mathrm{rs}}[[j, s]]-201 / 2\right)^{2}\right)},\{i, 1,18\}$,

$\{j, 1,18\}]$

$\operatorname{Do}\left[C 2[i, 19]=\left(\sum_{t=1}^{200}\left(\mathrm{IB}_{r s}[i, t]\right]-201 / 2\right) *\left(\mathrm{IB}_{\mathrm{Is}}[[19, t]]-201 / 2\right)\right) /$

$\left.\sqrt{ }\left(\left(\sum_{t=1}^{200}\left(\operatorname{LB}_{\mathrm{rs}}[[i, t]]-201 / 2\right)^{2}\right) *\left(\sum_{s=1}^{200}\left(\operatorname{RB}_{\mathrm{rs}}[[19, s]]-201 / 2\right)^{2}\right)\right),(i, 1,18\}\right]$

$\operatorname{Do}[C 2[19, i]=C 2[i, 19],\{i, 1,18\}]$

$C 2[19,19]=1$;

Unprotect $[C] ; C 2=\operatorname{Array}[C 2,\{19,19\}]$;

$B 2$ = Inverse $[\mathrm{C} / / \mathrm{N}]$;

$D O\left[\gamma B_{c s}[i]=-B 2[[i, 19]] / \sqrt{B 2[[i, i]] * B 2[[19,19]]} / / N,\{i, 1,14\}\right]$

EROCirputparamsandB $=T$ Table $\left[\gamma B_{1 s}[i],\{i, 1,14\}\right]$

$\{0.00978377,0.111723,0.986615,-0.158037,0.168761,-0.0427989,-0.0835763$,

$0.8786,-0.0692339,0.0598107,-0.0593243,0.0090944,-0.00995819,-0.104315\}$ 
$D o\left[t B_{r s}[i]=\gamma B_{r s}[i] * \sqrt{\frac{198}{1-\gamma B_{r s}[i] * \gamma B_{r s}[i]}} / / N,\{i, 1,14\}\right]$

tstatisticsB $B_{r s}=$ Table $\left[\operatorname{tB}_{r s}[i],\{i, 1,14\}\right]$

$\{0.137676,1.58199,85.1375,-2.25208,2.40923,-0.602786,-1.18015$,

$25.8879,-0.976551,0.843121,-0.83624,0.127975,-0.140131,-1.47589\}$

$\operatorname{Do}\left[\right.$ sigleve12[i] $=2-2 * \operatorname{CDF}\left[\right.$ StudentTDistribution [198], $\left.\operatorname{Sign}\left[t \mathrm{~B}_{\mathrm{rs}}[i]\right] * \mathrm{tB}_{\mathrm{rs}}[i]\right]$,

$\{i, 1,14\}]$

significancelevelsERCCSandB $B_{\mathrm{rs}}=$ Table $[\operatorname{siglevel2}[i],\{i, 1,14\}]$

$\{0.890636,0.115248,0 ., 0.0254148,0.0169018,0.547341$,

$0.239356,0 ., 0.329983,0.400178,0.404028,0.898299,0.888699,0.141561\}$

orderedfinaloutoome [3] = Sort [finaloutcome [3] ] ;

rankoolumn[19] $=$

Table [Position [orderedfinaloutocme[3], finaloutcome[3] [ [i] ]], \{i, 1, 200\}] // Flatten;

rankmatrixB $B_{s r}=$ Array $[$ rankoolumfunction, $\{200,19\}]$;

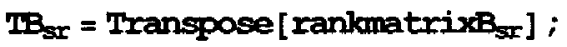

$D \circ\left[c 3[i, j]=\left(\sum_{t=1}^{200}\left(\mathbb{F B}_{s x}[[i, t]]-201 / 2\right) *\left(\mathrm{~TB}_{s x}[[j, t]]-201 / 2\right)\right) /\right.$

$\sqrt{\left(\sum_{t=1}^{200}\left(\mathrm{HB}_{\mathrm{gx}}[[i, t]]-201 / 2\right)^{2}\right) *\left(\sum_{s=1}^{200}\left(\mathrm{HB}_{\mathrm{sx}}[[j, s]]-201 / 2\right)^{2}\right)},\{i, 1,18\}$,

$\{j, 1,18\}]$

$D O\left[C 3[i, 19]=\left(\sum_{t=1}^{200}\left(\operatorname{TB}_{s x}[[i, t]]-201 / 2\right) *\left(\operatorname{TB}_{s x}[[19, t]]-201 / 2\right)\right) /\right.$

$\left.\sqrt{ }\left(\left(\sum_{t=1}^{200}\left(\operatorname{LB}_{s x}[[i, t]]-201 / 2\right)^{2}\right) *\left(\sum_{s=1}^{200}\left(\operatorname{LB}_{s x}[[19, s]]-201 / 2\right)^{2}\right)\right),\{i, 1,19\}\right]$

$\operatorname{Do}[03[19, i]=03[i, 19],\{i, 1,19\}]$

c3 $[19,19]=1$;

Urprotect $[C] ; C 3=\operatorname{Array}[C 3,\{19,19\}]$;

$\mathrm{B} 3=$ Inverse $[\mathrm{C} 3 / / \mathrm{N}]$;

$\left.D o\left[\gamma B_{s r}[i]=-B 3[[i, 19]] / \sqrt{B 3}[[i, i]] * B 3[119,19]\right] / / N,\{i, 1,14\}\right]$

EROCinputparamsandB $\mathrm{sr}=$ Table $\left[\gamma \mathrm{B}_{\mathrm{sr}}[i],\{i, 1,14\}\right]$

$\{0.0359044,0.872152,0.078262,-0.637174,-0.0144595,0.623413,0.0626986$,

$-0.521915,0.0302684,0.861561,0.0081576,-0.0734403,-0.036471,0.0749015$ \}

$D O\left[t B_{s x}[i]=\gamma B_{s I}[i] * \sqrt{\frac{198}{1-\gamma B_{s x}[i] * \gamma B_{s x}[i]}} / / N,\{i, 1,14\}\right]$

tstatisticsB $\mathrm{B}_{\mathrm{sr}}=$ Table $\left[\mathrm{tB}_{\mathrm{sr}}[i],\{i, 1,14\}\right]$

$\{0.505545,25.0846,1.10463,-11.6331,-0.203484,11.2192,0.883986$,

$-8.60963,0.426109,23.8809,0.114791,-1.0362,-0.513534,1.05693$ \} 


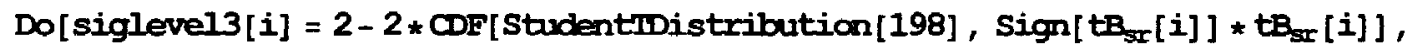

$\{i, 1,14\}]$

significancelevelsPROCSandB $=$ Table $[$ siglevel3 $[i],\{i, 1,14\}]$

$\{0.613738,0 ., 0.27066,0 ., 0.838966,0 ., 0.377776$,

$\left.2.22045 \times 10^{-15}, 0.670491,0 ., 0.908727,0.301375,0.608151,0.291833\right\}$

orderedfinaloutcome [4] = Sort [finaloutoome [4] ] ;

rankoolumn [19] =

Table [Position [orderedfinaloutoome [4], finaloutoome[4] [i]]] , \{i, 1, 200\}] // Flatten; rankmatrix $B_{n r}=$ Array [ rankoolumfunction, $\{200,19\}$ ] ;

$\mathbf{T B}_{\text {II }}=$ Transpose $\left[\right.$ rankmatrix $\left.\mathbf{B}_{\text {rI }}\right]$;

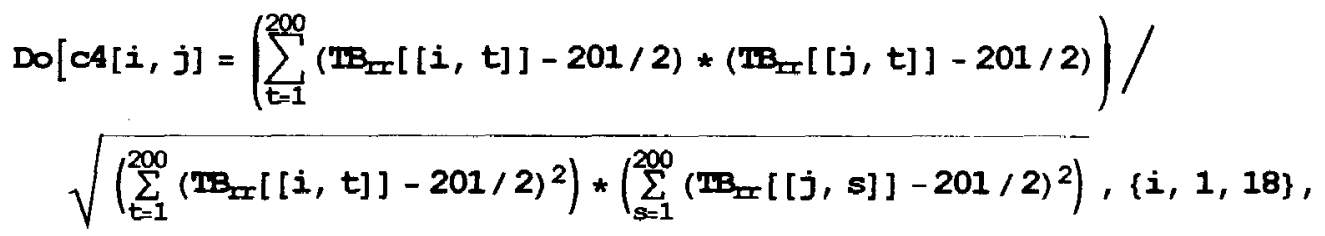

$\{j, 1,18\}]$

$D o\left[C 4[i, 19]=\left(\sum_{t=1}^{200}\left(\operatorname{TB}_{\operatorname{mrx}}[[i, t]]-201 / 2\right) *\left(\operatorname{TB}_{\operatorname{Ir}}[[19, t]]-201 / 2\right)\right) /\right.$

$\left.\sqrt{ }\left(\left(\sum_{t=1}^{200}\left(\operatorname{TB}_{1 x}[[i, t]]-201 / 2\right)^{2}\right) *\left(\sum_{s=1}^{200}\left(\operatorname{LB}_{1 x}[[19, s]]-201 / 2\right)^{2}\right)\right),\{i, 1,19\}\right]$

$\operatorname{Do}[c 4[19, i]=c[i, 19],\{i, 1,18\}]$

$C 4[19,19]=1$;

Urprotect $[C] ; C 4=$ Array $[C 4,\{19,19\}]$;

$\mathrm{B4}=$ Inverse $[\mathrm{C4} / / \mathrm{N}]$;

$D O\left[\gamma B_{ \pm I}[i]=-B 4[[i, 19]] / \sqrt{B 4[[i, i]]} * B 4[[19,19]] / / N,\{i, 1,14\}\right]$

FROCinputparamsandB $B_{I x}=$ Table $\left[\gamma B_{\text {Ir }}[i],\{i, 1,14\}\right]$

$\{0.00839003,0.110428,0.166616,-0.418732,-0.0184253,-0.0129835,0.409715$, $-0.688149,0.033012,0.121917,0.00430189,0.00198342,-0.00585949,0.0393947$ \}

$D o\left[t B_{I x}[i]=\gamma B_{x x}[i] * \sqrt{\frac{198}{1-\gamma B_{I x}[i] * \gamma B_{I x}[i]}} / / N,\{i, 1,14\}\right]$

tstatisticsB $B_{x x}=$ Table $\left[\operatorname{tB}_{x x}[i],\{i, 1,14\}\right]$

$\{0.118062,1.56343,2.37773,-6.4883,-0.259311,-0.182709,6.32001$,

$-13.3455,0.464773,1.72842,0.0605335,0.0279093,-0.0824517,0.554764\}$

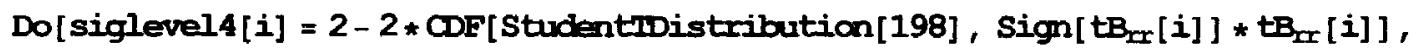
$\{i, 1,14\}]$

significancelevelsPRCCSandB $=$ Table $[$ siglevel4 $[i],\{i, 1,14\}]$

$\left\{0.906138,0.119549,0.0183716,6.80493 \times 10^{-10}, 0.795665,0.855213\right.$,

$\left.1.69641 \times 10^{-9}, 0 ., 0.642604,0.085471,0.951792,0.977763,0.934371,0.579682\right\}$ 


\section{APPENDIX 2}

\section{Calculation of Partial Rank Correlation Coefficients}

The following technique, adapted from [9], is used to calculate PRCC's between input variables and a single output variable. The process is repeated separately for each output variable.

Add outcome vector to the $N \times K$ LHS table as column $K+1$. Form a new $N \times(K+1)$ matrix by replacing each value in the original matrix by its rank. For each column, assign the smallest value in the column a rank of 1 , the next largest value a rank of 2 , continuing in this fashion until the largest value in the column is assigned a rank of $N$. If two parameters have identical ranks on every run of the model, then use only one of those parameters in calculating PRCC's. The new matrix has the form

$$
\left(\begin{array}{ccccc}
r_{11} & r_{21} & \cdots & r_{K 1} & R_{1} \\
r_{12} & r_{22} & \cdots & r_{K 2} & R_{2} \\
\vdots & \vdots & & \vdots & \vdots \\
r_{1 N} & r_{2 N} & \cdots & r_{K N} & R_{N}
\end{array}\right)
$$

where $r_{s t}=$ rank of $s^{\text {th }}$ input parameter on $t^{\text {th }}$ run of model and $R_{t}=$ rank of outcome variable on $t^{\text {th }}$ run of model $(s=1,2, \ldots, K ; t=1,2, \ldots, N)$.

Define the $(K+1) \times(K+1)$ symmetric correlation matrix $\mathrm{C}$, with elements $c_{i j}$, as follows:

$$
c_{i j}=\frac{\sum_{i=1}^{N}\left(r_{i t}-\frac{1+N}{2}\right)\left(r_{j t}-\frac{1+N}{2}\right)}{\sqrt{\sum_{t=1}^{N}\left(r_{i t}-\frac{1+N}{2}\right)^{2} \sum_{s=1}^{N}\left(r_{j s}-\frac{1+N}{2}\right)^{2}}}
$$


For the $c_{i, K+1}$ elements of $\mathrm{C}$, replace $r_{j t}$ and $r_{j s}$ with $R_{i}$. Elements on the main diagonal of $\mathrm{C}$ are all ones. The formula above is Pearson's product moment correlation coefficient (See Conover [64], p. 244). A discussion of the correlation matrix is included in Kendall $\&$ Stuart [65], pp. 338-339.

Let $\mathrm{B}=\left(b_{i j}\right)=\mathrm{C}^{-1}$. The outcome variable may be viewed as parameter $K+1$. The PRCC of the $i^{\text {th }}$ parameter with respect to the outcome variable is $\gamma_{i, K+1}$ :

$$
\begin{aligned}
\gamma_{, K+1} & =\frac{-\left(\text { cofactor of } c_{i, K}+1\right)}{\sqrt{\left(\text { cofactor of } c_{i i}\right)\left(\text { cofactor of } c_{i, K+1}\right)}} \\
& =\frac{-\left(\text { cofactor of } c_{i, K+1}\right)}{\sqrt{\left(\frac{\text { (cofactor of } \left.c_{i i}\right)}{\operatorname{det} \mathrm{C}}\right)\left(\frac{\left(\operatorname{cofactor} \text { of } c_{i, K+1}\right)}{\operatorname{det} \mathrm{C}}\right)}} \\
& =\frac{-b_{i, K+1}}{\sqrt{b_{i i} b_{K+1, K+1}}}
\end{aligned}
$$

Significance of nonzero PRCC's are determined with the statistic

$$
t_{i, K+1}=\gamma, K+1 \sqrt{\frac{N-2}{1-(\gamma, K+1)^{2}}}
$$

where the variable $\mathrm{t}$ is distributed in Student's t-distribution with $N-2$ degrees of freedom. 


\section{CURRICULUM VITAE}

NAME: $\quad$ Susan L. Calcote White

ADDRESS: Department of Mathematics

328 Natural Sciences Building

University of Louisville

Louisville, KY 40292

DOB: $\quad$ Lake Charles, Louisiana - July 31, 1971

EDUCATION

\& TRAINING: $\quad$ B.S., Secondary Mathematics Education McNeese State University

1989-1992 